\title{
p53 coordinates decidual sestrin 2/AMPK/mTORC1 signaling to govern parturition timing
}

\author{
Wenbo Deng, ${ }^{1}$ Jeeyeon Cha, ${ }^{1}$ Jia Yuan, ${ }^{1}$ Hirofumi Haraguchi, ${ }^{2}$ Amanda Bartos, ${ }^{1}$ Emma Leishman, ${ }^{3}$ Benoit Viollet, ${ }^{4,5,6}$ \\ Heather B. Bradshaw, ${ }^{3}$ Yasushi Hirota, ${ }^{1,2}$ and Sudhansu K. Dey ${ }^{1}$ \\ 'Division of Reproductive Sciences, Cincinnati Children's Hospital Medical Center, Cincinnati, Ohio, USA. ²Department of Obstetrics and Gynecology, Graduate School of Medicine, University of Tokyo, \\ Tokyo, Japan. ' ${ }^{3}$ Department of Psychological and Brain Sciences, Indiana University, Bloomington, Indiana, USA. ${ }^{4}$ Institut Cochin, INSERM U1016, Paris, France. ${ }^{5}$ CNRS, UMR 8104, Paris, France. \\ שUniversité Paris Descartes, Sorborne Paris Cité, Paris, France.
}

\begin{abstract}
Inflammation and oxidative stress are known risk factors for preterm birth (PTB); however, the mechanisms and pathways that influence this condition are not fully described. Previously, we showed that mTORC1 signaling is increased in mice harboring a uterine-specific deletion of transformation-related protein 53 ( $p 53^{d / d}$ mice), which exhibit premature decidual senescence that triggers spontaneous and inflammation-induced PTB. Treatment with the mTORC1 inhibitor rapamycin reduced the incidence of PTB in the $p 53^{d / d}$ mice. Decidual senescence with heightened mTORC1 signaling is also a signature of human PTB. Here, we have identified an underlying mechanism for PTB and a potential therapeutic strategy for treating the condition. Treatment of pregnant $p 53^{d / d}$ mice with either the antidiabetic drug metformin or the antioxidant resveratrol activated AMPK signaling and inhibited mTORC1 signaling in decidual cells. Both metformin and resveratrol protected against spontaneous and inflammation-induced PTB in $p 53^{d / d}$ females. Using multiple approaches, we determined that $p 53$ interacts with sestrins to coordinate an inverse relationship between AMPK and mTORC1 signaling that determines parturition timing. This signature was also observed in human decidual cells. Together, these results reveal that p53-dependent coordination of AMPK and mTORC1 signaling controls parturition timing and suggest that metformin and resveratrol have therapeutic potential to prevent PTB.
\end{abstract}

\section{Introduction}

Preterm birth (PTB) significantly accounts for neonatal morbidity and mortality, making PTB a major clinical, economic, and social concern in many parts of the world, including the United States (1). PTB is a syndrome culminating from many risk factors, including genetic predisposition, infection/inflammation, environmental factors, oxidative stress, progesterone $\left(\mathrm{P}_{4}\right)$ resistance, and extremes in maternal age (2). Surviving premature babies often suffer an array of serious chronic disabilities. Because of the complex nature of the disease, defining the mechanisms underlying PTB has proven difficult.

Animal models that are genetically predisposed to develop spontaneous PTB are powerful tools for studying underlying mechanisms and developing novel treatment strategies for this multifaceted disease. However, most animal studies often use systemic or local exposure to high doses of inflammatory agents, such as LPS, or cytokines that induce ovarian luteolysis with a decline in blood $\mathrm{P}_{4}$ levels and other systemic effects, resulting in PTB and/or embryonic resorptions (3). However, decline in blood $\mathrm{P}_{4}$ levels, which triggers parturition in mice, does not appear to occur in human parturition $(2,4)$. We generated a unique mouse model with conditional uterine deletion of tumor suppressor gene p53 (Trp53/f/fl $\mathrm{Pgr} \mathrm{Cre} /+$

Authorship note: W. Deng and J. Cha contributed equally to this work

Conflict of interest: The authors have declared that no conflict of interest exists.

Submitted: March 23, 2016; Accepted: May 24, 2016.

Reference information: J Clin Invest. 2016;126(8):2941-2954. doi:10.1172/JCI87715. mice, referred to here as $p 53^{d / d}$ mice), and we showed that uterine deficiency of $\mathrm{p} 53$ confers premature decidual senescence and elicits spontaneous PTB without a fall in $\mathrm{P}_{4}$ levels, thereby introducing a model to study human parturition $(5,6)$. These studies found that increased mTORC1 signaling caused premature decidual senescence during early pregnancy, with $50 \%$ to $60 \%$ of $p 53^{d / d}$ females showing spontaneous PTB with increases in COX2-derived prostaglandins (PGs). Rapamycin, an inhibitor for mTORC1 signaling, or a COX2 inhibitor, celecoxib, prevented PTB in $p 53^{d / d}$ females $(5,6)$. PTB in these genetically predisposed mice was greatly aggravated with exposure to even a low dose of ultrapure LPS $(10 \mu \mathrm{g})$ with a decline in $\mathrm{P}_{4}$ levels; this dose of LPS had no apparent adverse effects on the floxed $p 53$ dams ( $p 53^{f / f l}$ dams) or their fetuses. When rapamycin and $\mathrm{P}_{4}$ were combined and used as a treatment, the incidence of PTB in $p 53^{d / d}$ females given LPS was dramatically reduced (4). These results suggested that cooperative contributions from both the decidua and ovary are critical for parturition timing and pregnancy success. A similar signature of decidual senescence with increased mTORC1 signaling and COX2 expression was noted in a subset of women undergoing PTB (4). Therefore, these findings of heightened mTORC1 signaling and decidual aging via cellular senescence are likely to have a significant impact on understanding and treating women undergoing PTB.

The above findings piqued our interest to further explore the mechanism underlying parturition timing and to search for more clinically acceptable drugs to target decidual aging and prevent PTB in $p 53^{d / d}$ females. With more than 50 years of clinical use, 
metformin (Met) is widely used as a first-line therapy for diabetes to increase insulin sensitivity $(7,8)$. Its off-label use in ovulation induction in women with polycystic ovary syndrome is supported by its safety reports in pregnancy $(9,10)$. Met can inhibit mTORC1 through an AMP-activated protein kinase-dependent (AMPK-dependent) pathway by altering the AMP/ATP ratio (11, 12) or through AMPK-independent pathways (13). Recent reports also show Met's potential use as an anti-aging therapy (14); it is currently under a clinical trial to explore its anti-aging effects (15). Therefore, we speculated that Met would be a viable option to reduce the incidence of PTB. In the same vein, resveratrol (Rsv), a natural derivative of grapes, was reported to have antioxidant and anti-aging properties with health benefits, including protection from heart disease and obesity, as well as improvement of mitochondrial function $(16,17)$. However, Rsv's definitive mechanism of action remains under investigation (18). Therefore, we asked whether Met or Rsv would mitigate PTB in $p 53^{d / d}$ mice by altering AMPK and mTORC1 signaling without adverse effects on the mother and pups.

We show here that Met or Rsv fed intermittently was highly effective in rescuing spontaneous PTB in $p 53^{d / d}$ mothers, and substantially reduced the incidence of PTB in $p 53^{d / d}$ females exposed to LPS $(10 \mu \mathrm{g})$ following a $\mathrm{P}_{4}$ injection. Further investigation found that these drugs execute their beneficial effects by forging an inverse relationship between AMPK and mTORC1 signaling to reduce PTB rates by attenuating decidual senescence. There is evidence that sestrin 2 (SESN2), a transcriptional target of p53, provokes AMPK activation and dampens mTORC1 signaling in human cell lines (19-21). We also found direct regulation of SESN2 by $\mathrm{p} 53$ in mouse decidual cells and found that both mouse and human decidual cells are coordinated by p53-sestrin interactions to establish an inverse relationship between decidual AMPK and mTORC1 activities. These preclinical studies using mouse models and human decidual cells have generated new knowledge and insight in parturition biology that could provide a basis for therapeutics already being used in the treatment of specific pregnancy disorders to combat human PTB.

\section{Results}

Met or Rsv reduces the incidence of PTB in $p 53^{d / d}$ females. Premature decidual senescence is a major cause of PTB in $p 53^{d / d}$ females (2). The spontaneous PTB rate in $p 53^{d / d}$ females ranges between $50 \%$ and $60 \%$. This could be due to other compensatory mechanisms or individual variation in sensitivity to genetic disposition to PTB. Thus, when this genetic disposition experiences even a small inflammatory insult, $100 \%$ of $p 53^{d / d}$ mothers show PTB (4). Since Met and Rsv are reported to attenuate cellular aging, increase lifespan, and inhibit mTORC1 activity with safe pharmacological profiles in pregnancy $(9,17,22)$, we assessed the efficacy of Met or Rsv on parturition timing in $p 53^{d / d}$ mice. $p 53^{f / f l}$ and $p 53^{d / d}$ mice were given Met or Rsv during pregnancy (Figure 1, A and B), and parturition timing was assessed as previously described (4). Met given by oral gavages $(1 \mathrm{mg} / \mathrm{kg} \mathrm{BW})$ on days 8,10 , and 12 remarkably decreased the rate of spontaneous PTB from $50 \%$ to only $9 \%$ in $p 53^{d / d}$ mothers (Figure $1 C$ ). Similarly, Rsv administered by oral gavages (30 mg/kg BW) on days $8,10,12$, and 14 reduced the spontaneous PTB rate from $50 \%$ to only $10 \%$ in $p 53^{d / d}$ mice
(Figure 1C). Concurrently, the number of live pups born to $p 53^{d / d}$ females after treatment with Met or Rsv substantially increased (Figure 1D). The treatments with Met and Rsv did not appear to exert detrimental effects on pregnancy outcomes in littermates of $p 53^{f / f l}$ dams (Figure 1D), and no drop in $\mathrm{P}_{4}$ levels was noted in $p 53^{f / f l}$ and $p 53^{d / d}$ mice (Supplemental Figure 1; supplemental material available online with this article; doi:10.1172/JCI87715DS1). The effective regimen and dose of Met and Rsv were determined from our initial pilot experiments and previous studies $(17,23)$. Higher doses of Met (30-300 mg/kg BW) have been reported in prior metabolic studies in mice $(11,24)$.

Inflammation has been widely used to trigger PTB in several rodent models using doses as high as $200 \mu \mathrm{g}$ of LPS by various routes of administration (3). We have shown that a low-grade inflammatory insult (10 $\mu \mathrm{g}$ LPS) superimposed on genetic predisposition incurs PTB in $100 \%$ of $p 53^{d / d}$ females. This is primarily due to suboptimal decidual health with a concomitant drop in $\mathrm{P}_{4}$ levels secondary to ovarian luteolysis in $p 53^{d / d}$ mothers. In contrast, $p 53^{f / f l}$ pregnant females showed no recognizable adverse effects with this low dose of LPS (4). To assess the efficacy of Met or Rsv in this context, LPS (10 $\mu \mathrm{g} /$ mouse) was injected i.p. in mice pretreated with Met or Rsv on day 16 (Figure 1, A and B). Met or Rsv alone could not deter the incidence of PTB in LPS-treated $p 53^{d / d}$ mothers (Figure 1E). However, a single injection of $\mathrm{P}_{4}(1-2$ $\mathrm{mg}$ /mouse) given 4 hours before LPS injection in Met- or Rsv-pretreated $p 53^{d / d}$ mice was able to substantially reduce the rate of PTB (Figure 1E). Interestingly, when $\mathrm{P}_{4}$ alone was tested, $80 \%$ of treated dams still experienced PTB, as compared with $20 \%$ and $36 \%$ in Met- or Rsv-pretreated $p 53^{d / d}$ mothers, respectively, following an injection of $\mathrm{P}_{4}$ (Figure 1E), suggesting effective targeting of both decidual health and compensating ovarian luteolysis. The number of live pups born also significantly increased in Met- or Rsv-pretreated mice with $\mathrm{P}_{4}$ prior to an LPS injection (15\% vs. $57 \%$ and $32 \%$, respectively) in $p 53^{d / d}$ mothers (Figure $1 \mathrm{~F}$ ). $\mathrm{P}_{4}$ injection alone showed some adverse effects on pup viability in LPS-treated $p 53^{f / f l}$ females (Figure 1F); this is not surprising since $\mathrm{P}_{4}$ levels normally start declining approaching parturition in floxed mice (5). Dystocia phenotype (difficult delivery) seen in $p 53^{d / d}$ mothers was also improved by Met and Rsv treatment. Overall, the results suggest that both Met and Rsv are effective in reducing the incidence of PTB in genetically predisposed mice, although Met appears to be more effective in reducing the incidence of PTB than Rsv, even at a much lower dose.

Met treatment improves decidual health. The decidua serves as a transient signaling hub that directs parturition timing (6). We previously reported that compromised decidual health and function in $p 53^{d / d}$ females are reflected in premature decidual senescence, increased rates of spontaneous PTB, and highly increased response to a small dose of LPS (4). To assess the effects of Met treatment on decidual health, we first examined the status of $\gamma \mathrm{H} 2 \mathrm{AX}$ associated with DNA damage followed by staining of senescence-associated $\beta$-galactosidase (SA- $\beta$-gal), 2 markers of decidual senescence (25), on day 16 in $p 53^{d / d}$ mice pretreated with Met. Met treatment substantially reduced the number of $\gamma \mathrm{H} 2 \mathrm{AX}-$ positive decidual cells and intensity of SA- $\beta$-gal staining in $p 53^{d / d}$ mice (Figure 2, A and B). Deciduae in $p 53^{d / d}$ mice are normally much thinner because of premature and progressive senescence, 
A Scheme of metformin treatment
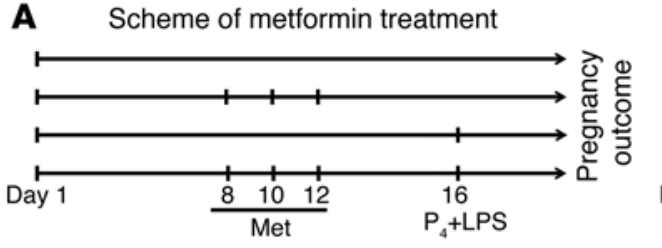

B

Scheme of resveratrol treatment
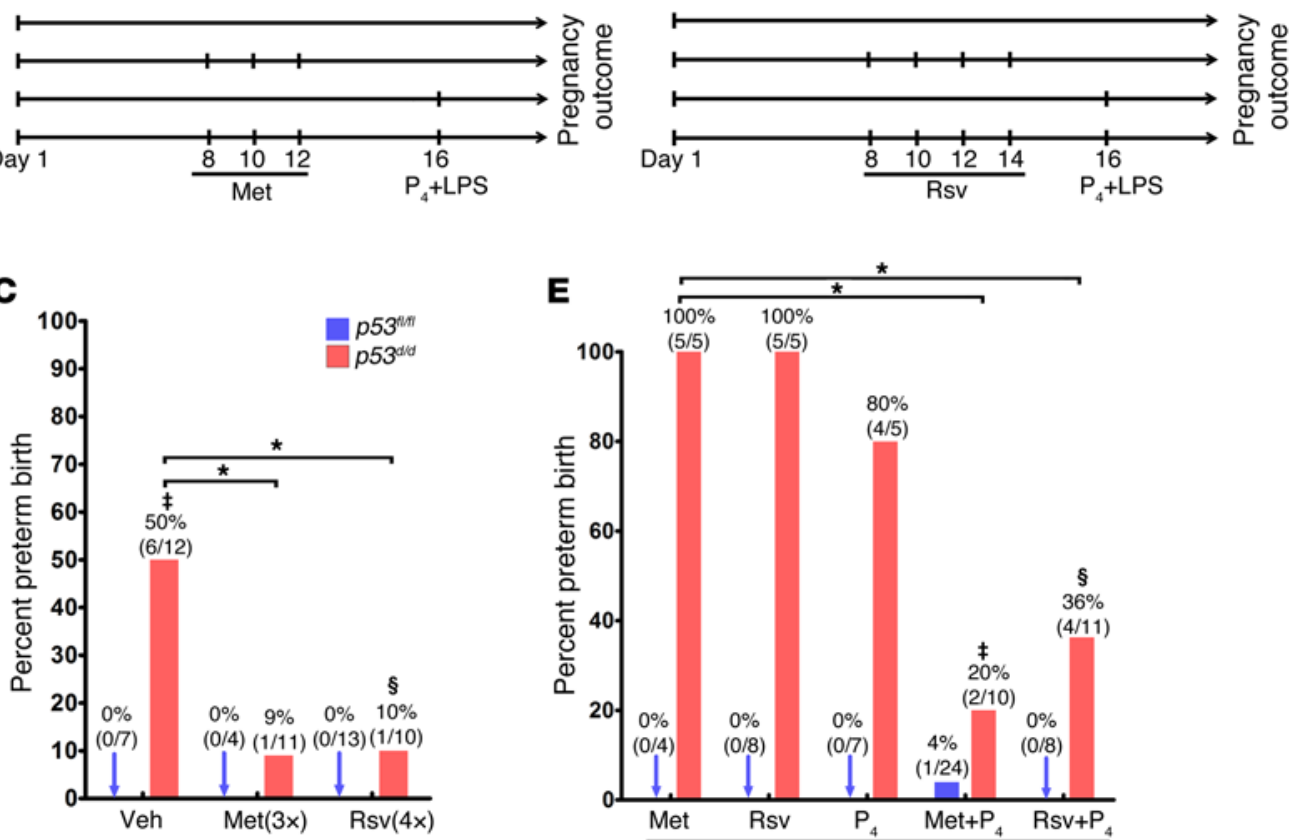

D

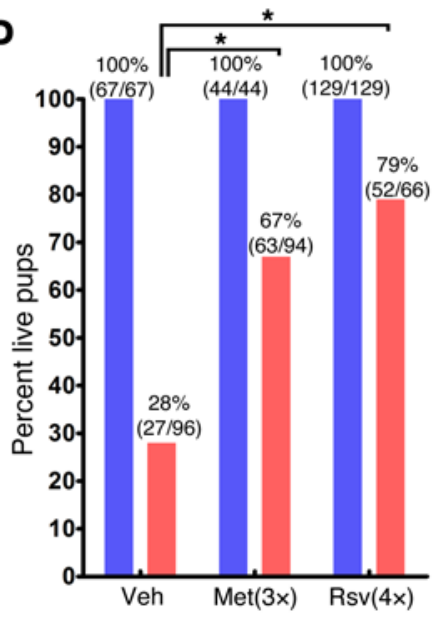

E

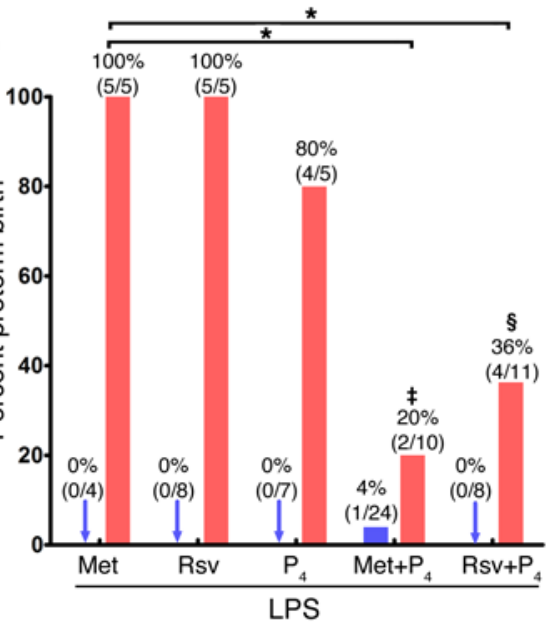

$\mathbf{F}$

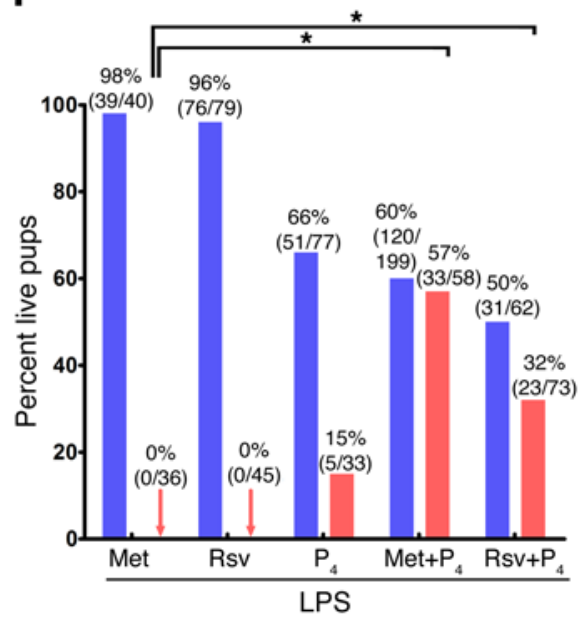

Figure 1. Met and Rsv attenuate preterm birth and dystocia. (A and B) Treatment schedules for Met and Rsv treatment. (C and D) Percent preterm birth and live pups under Met or Rsv treatment in $p 53^{f / f l}$ and $p 53^{d / d}$ females. ${ }^{\ddagger}$ Three of $\operatorname{six} p 53^{d / d}$ females that had preterm birth also showed dystocia. \$One of ten $p 53^{d / d}$ females showed both preterm birth and dystocia. ${ }^{*} P<0.05, \chi^{2}$ test. (E and F) Percent preterm birth and live pups under Met or Rsv in combination with $\mathrm{P}_{4} 4$ hours before an LPS $(10 \mu \mathrm{g})$ injection in $p 53^{f / f l}$ and $p 53^{d / d}$ mice. ${ }^{\ddagger}$ One of two $p 53^{d / d}$ females that had preterm birth also showed dystocia. ${ }^{\$}$ One of four $p 53^{d / d}$ females showing preterm birth also had dystocia. ${ }^{*} P<0.05, \chi^{2}$ test. but decidual thickness was significantly improved in Met-treated $p 53^{d / d}$ females (Figure 2B). Other indicators of decidual health were also assessed: prolactin receptor (Prlr) expression was examined by in situ hybridization and real-time PCR. Met treatment increased the signal intensity in both $p 53^{f / f l}$ and $p 53^{d / d}$ deciduae, though it was initially lower in $p 53^{d / d}$ decidua (Figure $2 \mathrm{C}$ ). Realtime PCR results more or less agree with in situ hybridization results (Supplemental Figure 2A). Blood vessel density, as determined by PECAM staining, increased in day $16 p 53^{d / d}$ deciduae after Met treatment (Supplemental Figure 2B).

Met upregulates AMPK signaling in $p 53^{d / d}$ deciduae. The above results led us to explore the mechanism by which these drugs show clear pregnancy benefits in a genetically predisposed mouse model. AMPK is considered an energy/nutrient-sensing hub on which Met exerts its influence (11). Since cell energetics is tightly linked to cell lifespan, and since both Met and Rsv have been shown to alter AMPK signaling $(26,27)$, we asked whether PTB could be influ- enced by AMPK signaling. We performed immunofluorescence (IF) localization of phosphorylated AMPK (pAMPK), as an index of AMPK activation, in $p 53^{f / f l}$ and $p 53^{d / d}$ deciduae on day 16 of pregnancy with or without Met treatment. We found that IF intensity of pAMPK was much lower in $p 53^{d / d}$ decidua compared with that in floxed littermates (Figure 2D). Met treatment increased the IF intensity of pAMPK in $p 53^{d / d}$ decidua (Figure 2D), but did not show much effect in $p 53^{f / f l}$ deciduae with already higher $\mathrm{pAMPK}$ levels. The decreases in PAMPK and its downstream target pRAPTOR (28) were associated with increased pS6 levels (mTORC1 signaling) in p53-deleted deciduae as evident from Western blotting results (Figure 2, E and F). Taken together, the results suggest that Met mitigates premature progression of decidual senescence by increasing pAMPK levels with decreased pS6 levels.

Met treatment attenuates $P G E_{2}$ and $P G F_{2 \alpha}$ levels in combination with $P_{4}$ in $p 53^{d / d}$ deciduae. PTB in $p 53^{d / d}$ dams is associated with increased decidual COX2 expression and $\mathrm{PGF}_{2 \alpha}$ levels on day 16 
A

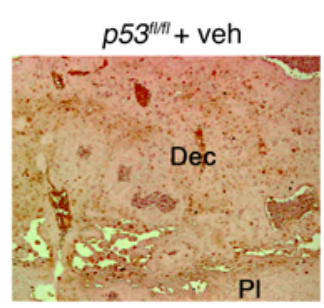

$\gamma \mathrm{H} 2 \mathrm{AX}($ day 16)

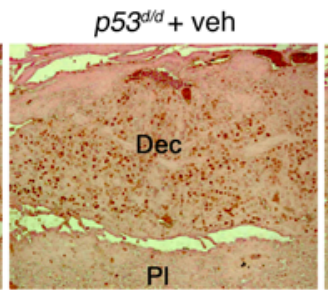

$p 53^{t / n t}+$ Met

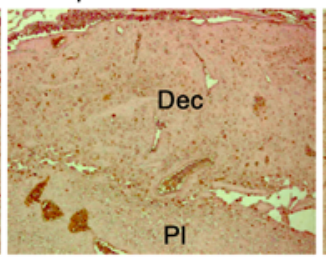

$p 53^{d / d}+$ Met

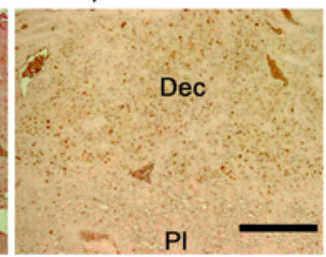

B

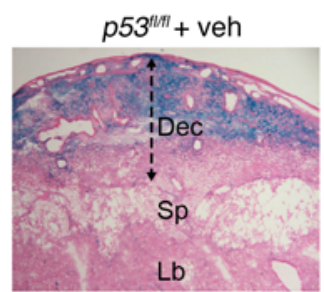

SA- $\beta$-gal staining (day 16)
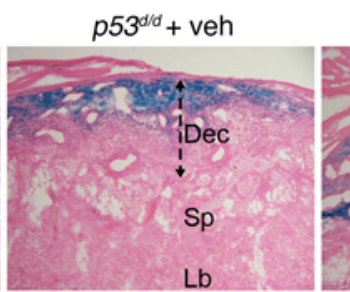

$p 53^{\text {thI }}+$ Met

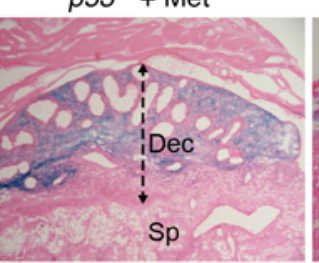

$p 53^{d / d}+$ Met

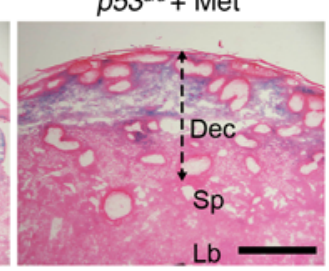

Figure 2. Met treatment improves decidual health. (A) Met reduced the number of $\gamma \mathrm{H} 2 \mathrm{AX}$-positive cells in $p 53^{d / d}$ deciduae. Scale bar: $250 \mu \mathrm{m}$. (B) Met attenuated decidual senescence in $p 53^{d / d}$ females. Scale bar: $500 \mu \mathrm{m}$. (C) Met upregulated Prlr expression in $p 53^{d / d}$ deciduae. Scale bar: 500 $\mu \mathrm{m}$. (D) PAMPK localization is low in $p 53^{d / d}$ deciduae and increased after Met treatment. Scale bar: $250 \mu \mathrm{m}$. bv, blood vessel; Dec, decidua; Lb, labyrinth; Sp, spongiotrophoblast. (E and F) pAMPK, pRAPTOR, and pS6 expression and quantitation in day 16 deciduae. pAMPK and pRAPTOR levels were lower in $p 53^{d / d}$ females with increased pS6 levels. Scale bar: $500 \mu \mathrm{m} .{ }^{*} P<0.05$, mean \pm SEM, Student's $t$ test.
C

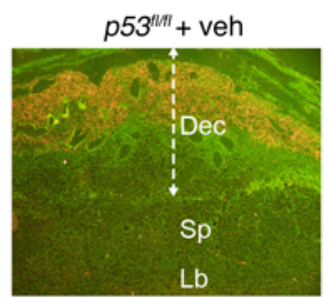

D

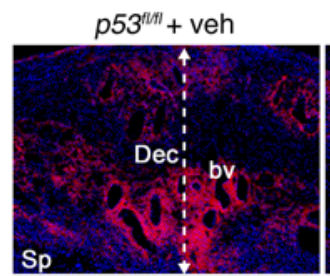

$\mathrm{pAMPK} / \mathrm{Nu}$

In situ hybridiation of Prlr in deciduae (day 16)

$p 53^{d / d}+$ veh

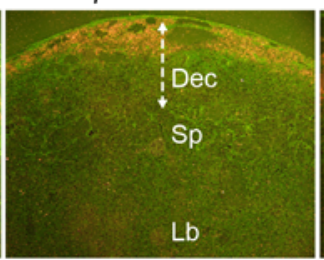

$p 53^{\text {thI }}+$ Met

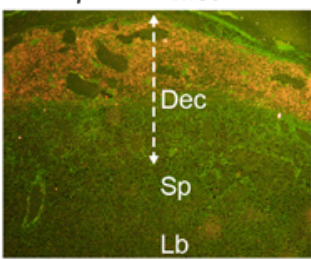

p $53^{d / d}+$ Met

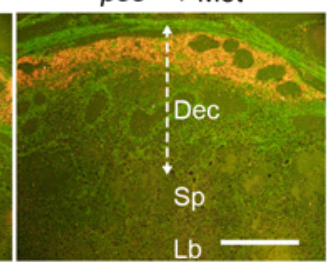

pAMPK localization in deciduae (day 16)

$p 53^{d / d}+$ veh

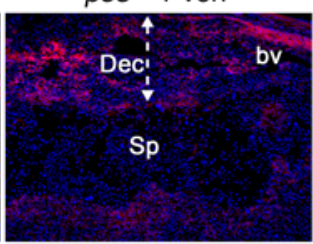

$p 53^{|t| r \mid}+$ Met

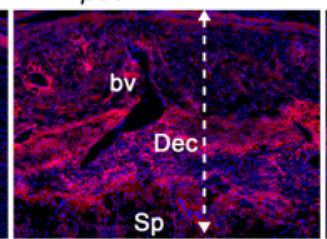

Sp

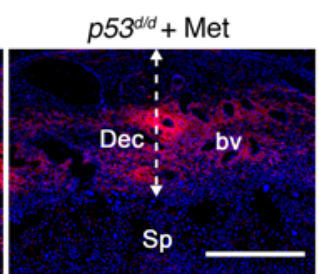

Sp
$\mathbf{E}$

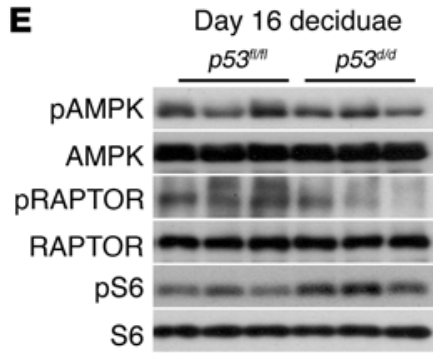

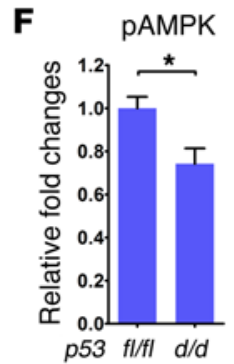

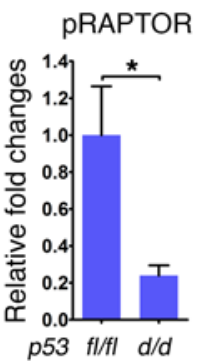

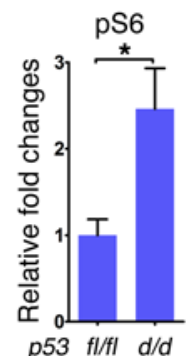

to trigger myometrial contractility (5). In addition to reducing the rate of PTB in $p 53^{d / d}$ mice, Met treatment downregulated the decidual COX2 expression as examined by in situ hybridization (Figure 3A). Since prostaglandins (PGs) are known to participate in parturition, we measured PG levels by high-performance liquid chromatography (HPLC) coupled with tandem mass spectrometry. We found that an injection of LPS $(10 \mu \mathrm{g})$ on day 16 significantly increased $\mathrm{PGF}_{2 \alpha}$ levels in $p 53^{d / d}$ deciduae without affecting the levels in $p 53^{f / f l}$ females; no changes in $\mathrm{PGE}_{2}$ levels were noted in $p 53^{f / f l}$ or $p 53^{d / d}$ deciduae. Met treatment alone did not reduce the levels of $\mathrm{PGF}_{2 \alpha}$ in $p 53^{d / d}$ females (Figure 3, B and C). However, Met treatment combined with an injection of $\mathrm{P}_{4}$ reduced the levels of $\mathrm{PGF}_{2 \alpha}$ and $\mathrm{PGE}_{2}$ in $p 53^{d / d}$ deciduae exposed to LPS (Figure 3, $\mathrm{B}$ and $\mathrm{C})$. These results are consistent with our findings of Met's inability to prevent PTB alone as opposed to substantial protection against PTB if Met treatment is combined with $\mathrm{P}_{4}$ after LPS exposure. Notably, Met reduced $\mathrm{PGF}_{2 \alpha}$ and $\mathrm{PGE}_{2}$ levels in both $p 53^{f / f l}$ and $p 53^{d / d}$ decidual cells in vitro, while Rsv decreased $\mathrm{PGE}_{2}$ 
A
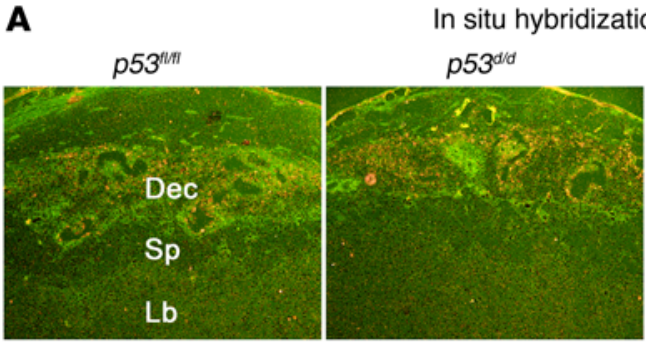

In situ hybridization of Cox2 (day 17)

$p 53^{\text {tunt }}+$ Met
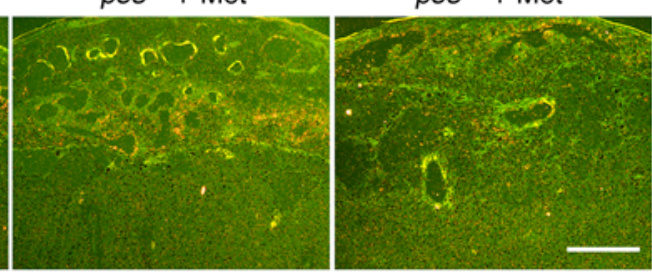

B Decidual PG levels (day 17)
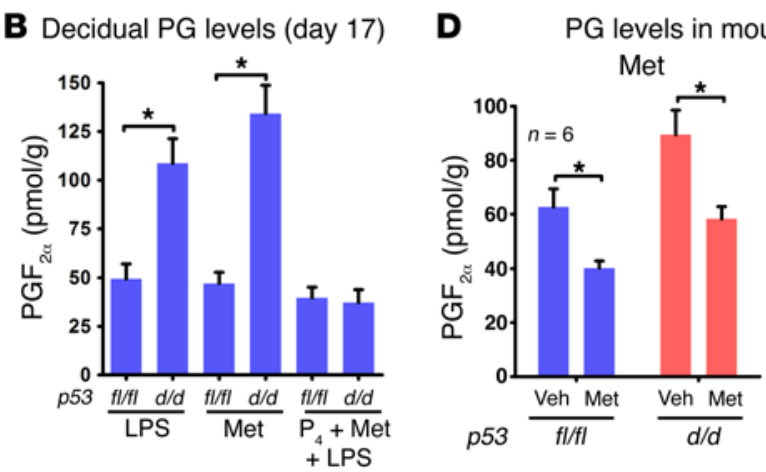

use stromal cells

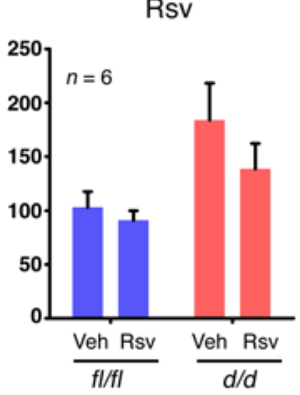

E
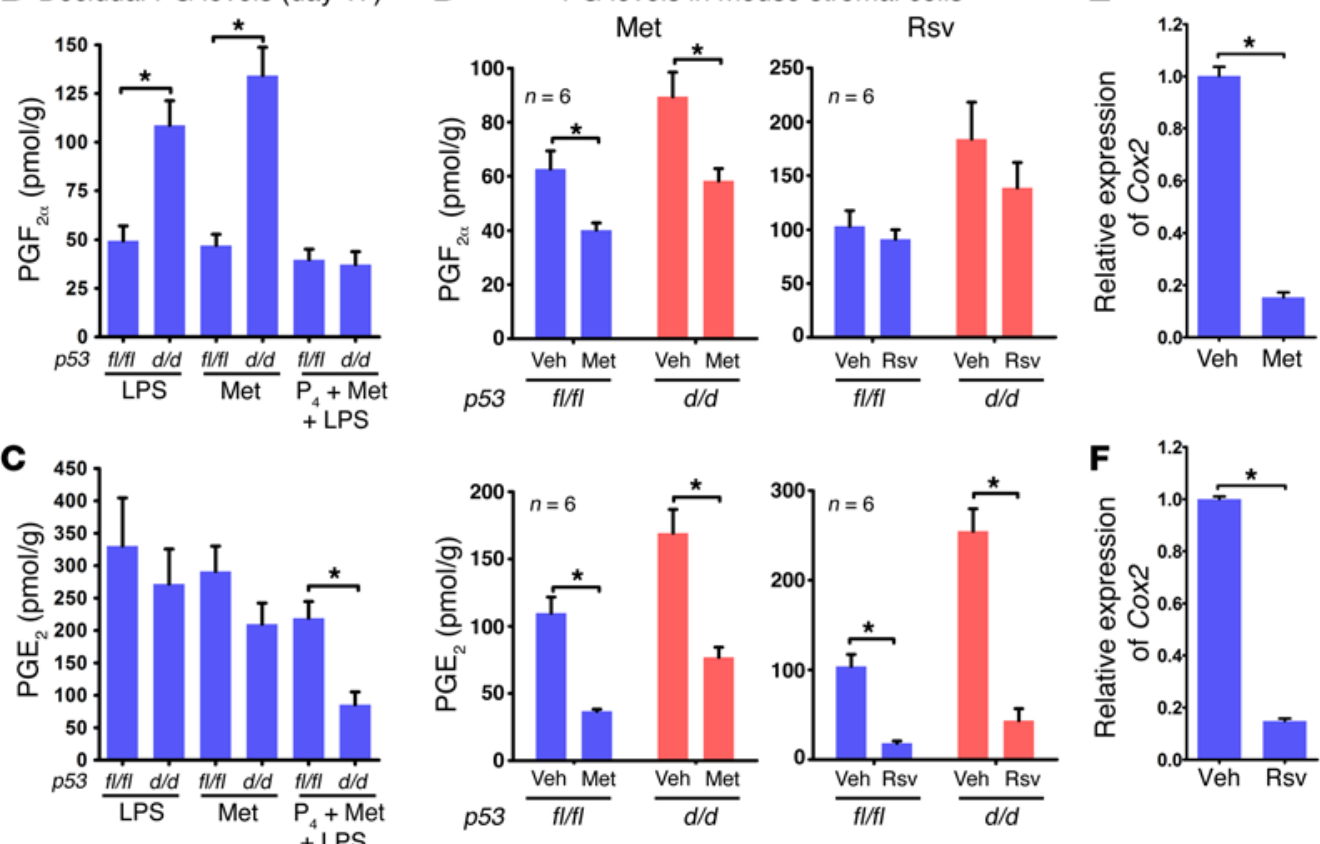
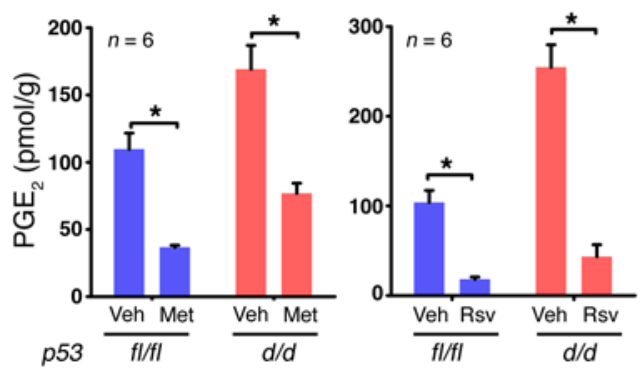

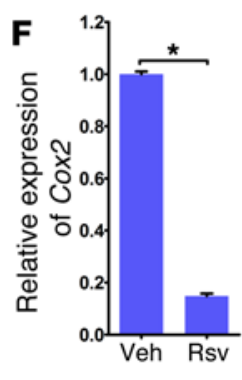

Figure 3. Met and Rsv regulate COX2-derived PG synthesis. (A) Upregulated Cox2 in $p 53^{d / d}$ deciduae was inhibited by Met. Dec, decidua; Lb, labyrinth; Sp, spongiotrophoblast. Scale bar: $500 \mu \mathrm{m}$. (B and C) Decidual PC levels on day 17 after Met treatment. PGF levels were higher in $p 53^{d / d}$ than in $p 53^{f / f l}$ mice after LPS injection, and levels were suppressed by combined treatment with Met and $\mathrm{P}_{4}\left(n=6,{ }^{*} P<0.05\right.$, mean $\pm \mathrm{SEM}$, Student's $t$ test). (D) PG levels in decidualized stromal cells after treatment with Met or Rsv. Met reduced $\mathrm{PGF} \mathrm{F}_{2 \alpha}$ levels in both $p 53^{f / / f l}$ and $p 53^{d / d}$ decidualized stromal cells, while Rsv had little effect. Met or Rsv inhibited PGE, levels in $p 53^{f / / f l}$ and $p 53^{d / d}$ decidualized stromal cells $\left(n=6,{ }^{*} P<0.05\right.$, mean \pm SEM, Student's $t$ test). (E and F) Met and Rsv attenuated Ptgs2 (encoding COX2) mRNA levels in decidualized mouse stromal cells ( $n=3,{ }^{*} P<0.05$, mean \pm SEM, Student's $t$ test). levels with limited effects on PGF PGel $_{2 \alpha}$ levels in both $p 53^{f l / f l}$ and $p 53^{d / d}$ decidual cells (Figure 3D). These results were reflected in lower Ptgs2 (encoding COX2) expression in decidual cells with Met or Rsv treatment (Figure 3, E and F). Similar inhibition of PTGS2 (encoding COX2) expression was also noted in human decidual cells exposed to Rsv (Supplemental Figure 3).

Met or Rsv inversely regulates AMPK and mTORC1 signaling in decidual cells. Given that Met or Rsv treatment improves decidual health by attenuating senescence and reduces PTB rates in $p 53^{d / d}$ mice, we explored the underlying mechanism by developing in vitro models of mouse and human decidualization and designed experiments to explore the molecular relationship between AMPK and mTORC1 signaling in the presence of Met or Rsv. WT mouse uterine stromal cells were isolated from day 4 pregnant mice and induced to decidualize in culture by hormone treatments for 4 days (29). Differentiation of stromal cells to decidual cells was confirmed by highly upregulated expression of prolactin family 8, subfamily a, member 2 (Prl8a2), a decidual marker in mice (ref. 30 and Supplemental Figure 4). Decidual cells were then cultured in the presence or absence of different concentrations of Met or Rsv to identify the lowest effective concentration. We found that Met at a dose of $10 \mathrm{mM}$ significantly increased the levels of pAMPK after 24 hours (Figure 4, A and B). This was associated with concomitant decreases in pS6 levels, an index of mTORC1 activity; Met even at a dose of $1 \mathrm{mM}$ downregulated pS6 levels (Figure 4, A and B).

We then asked as to why the dose of Met we used in decidualized stromal cells in vitro was so much higher than the dose used in vivo. Although several reasons, including differences in drug metabolic rate, uptake, and efficacy between in vivo and in vitro systems, could account for this observation, we think this discrepancy is perhaps due to differential expression of Met transporters in vivo and vitro. Met is transported inside the cell primarily by organic cationic transporters of the solute carrier family 22: SLC22A1 (OCT1) and SLC22A3 (OCT3) (31). Indeed, we found that while expression levels of Oct1 and Oct 3 were much higher in the liver followed by intestine and decidua in vivo, their expressions were much lower, but present, in decidual cells differentiated in vitro from stromal cells. This would suffice as to why a much higher concentration of Met is required to be effective in cultured cells (Supplemental Figure 5). In the same context, Rsv at different concentrations also upregulated pAMPK levels with downregulation of pS6 (Figure 4, C and D).

Other downstream targets of mTORC1 signaling, including HIF1 $\alpha$, pS6K (Thr389), p-eIF4E (Ser209), p4EBP1 (Thr70), p4EBP1 (Ser65), and p4EBP1 (Thr37/46), were also examined after Met or Rsv exposure in decidual cells in vitro. 4EBP1 upon phosphorylation serves as an activator of translation by releasing eIF4E 
A
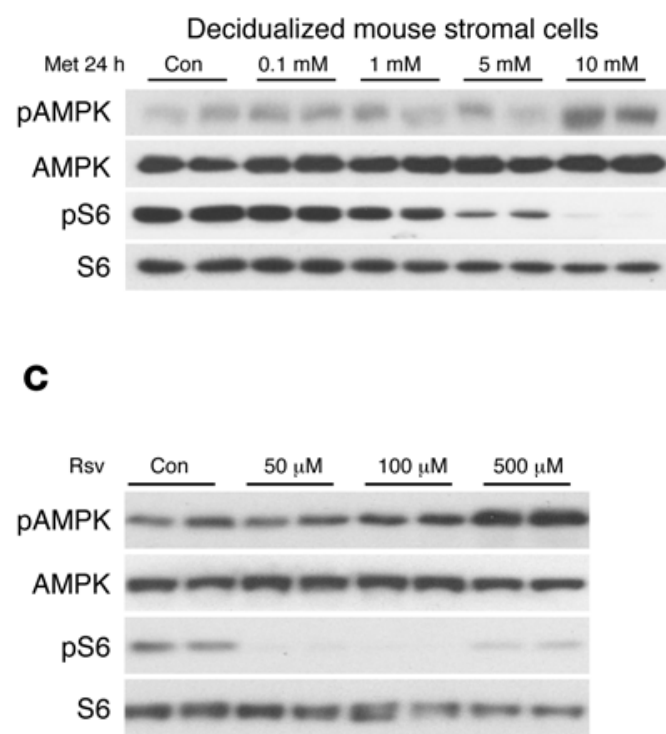

$\mathbf{E}$

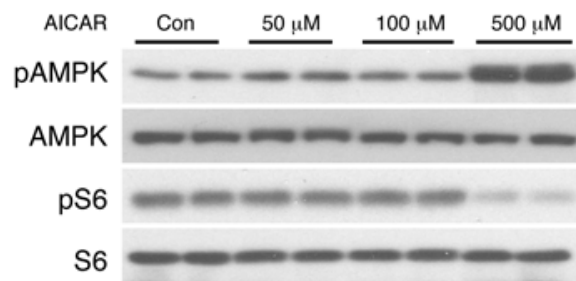

B

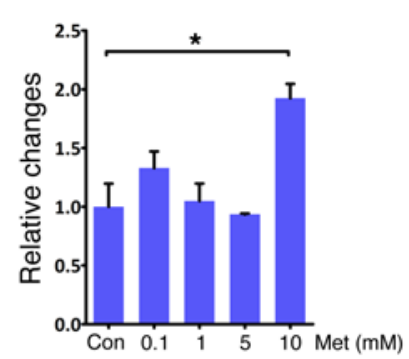

D

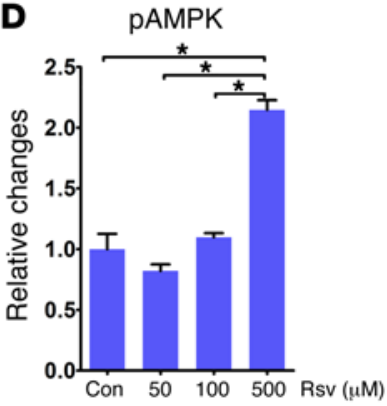

$\mathbf{F}$

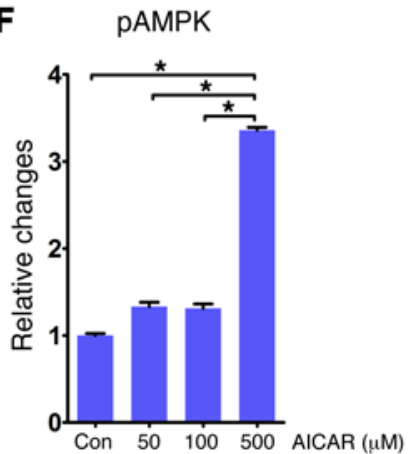

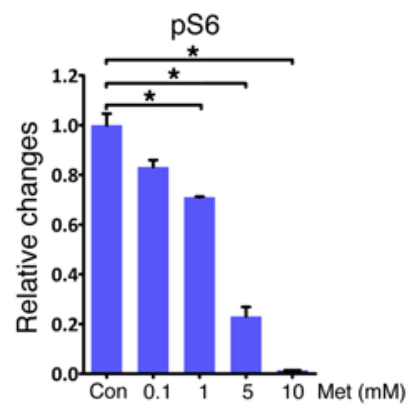
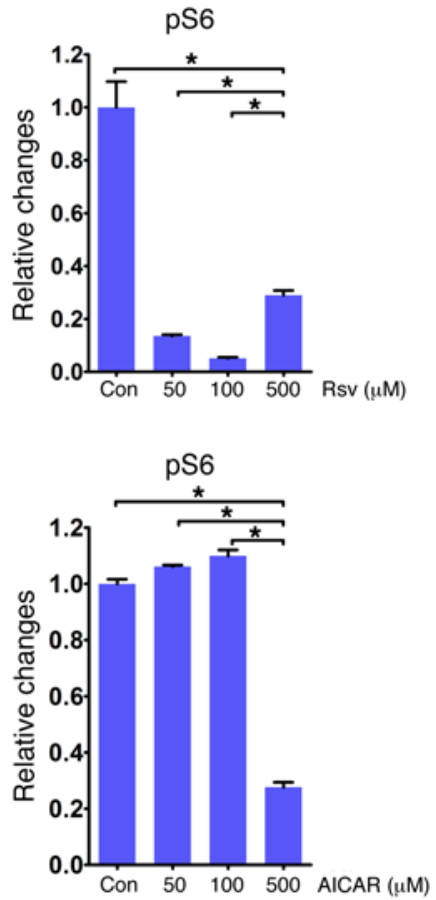

Figure 4. Met, Rsv, and AICAR affect pAMPK and pS6 levels in a concentration-dependent manner. (A and B) pAMPK levels and quantitation were significantly upregulated by Met $(10 \mathrm{mM})$ with reduced pS6 levels. (C and D) Levels of pAMPK and pS6 and quantitation were altered at 50, 100, and 500 $\mu \mathrm{M}$ by Rsv. (E and $\mathbf{F}$ ) Levels of pAMPK and pS6 and quantitation were significantly altered at $500 \mu \mathrm{M}$ AICAR. ${ }^{*} P<0.05$, mean \pm SEM, ANOVA.

(32). We found that all of these downstream mediators were downregulated by Met or Rsv treatment of decidual cells (Supplemental Figure 6, A-D). These results provide credence to the robustness of the mTORC1 signaling and its relationship with AMPK activity.

We further evaluated the interaction between mTORC1 and pAMPK signaling in decidualized stromal cells due to their inverse relationship in the presence of Met. Although both Met and rapamycin significantly inhibited pS6 activity in decidualized stromal cells, we found that rapamycin failed to alter the levels of pAMPK, suggesting that AMPK is upstream of the mTORC1 signaling pathway in decidual cells (Figure 5, A and B), as shown previously in other systems (33). Interestingly, 5-AICAR (aminoimidazole-4-carboxamide-1 $\beta$-D-ribofuranoside), an activator of AMPK (34), upregulated the levels of PAMPK concomitantly with decreases in pS6 levels (Figure 4, E and F), suggesting that AMPK activation would attenuate $\mathrm{mTORC} 1$ signaling. Collectively, these results show that Met or Rsv coordinates an inverse relationship between AMPK and mTORC1 signaling in decidualized mouse stromal cells, thus slowing decidual senescence against PTB.
Met or Rsv confers an inverse relationship between AMPK and $m$ TORC1 signaling in $p 53^{d / d}$ decidual cells. To understand the intricacies of AMPK and MTORC1 interactive regulation, $p 53^{f / f l}$ and $p 53^{d / d}$ uterine stromal cells were decidualized in vitro and treated with Met or Rsv; these treated cells showed increases in PAMPK levels with downregulation of pS6 levels (Figure 5, C-F). Consistent with our in vivo data (Figure 2D), baseline pAMPK levels were lower in primary $p 53^{d / d}$ decidualized cells than in floxed stromal cells (Figure 5, C-F). To further explore the relationship of $\mathrm{p} 53$ with $\mathrm{pAMPK}$ and mTORC1 signaling, we used $p 53^{-/-}$mouse embryonic fibroblast cells (MEFs), which have higher pS6 levels than the parental cell line (Figure 5, G and H); this could be due to TSC 2 as a direct target of p53 as evident from p53 enrichment in the TSC2 gene body as reported in published ChIP sequencing (ChIP-seq) data (35-37). p53-/- MEFs treated with Met showed increased pAMPK and decreased pS6 levels. These results again highlight a molecular interplay between AMPK and mTORC1 signaling and provide evidence for the contributory role of Met or Rsv toward protection against PTB in $p 53^{d / d}$ mice. Given the above 
A
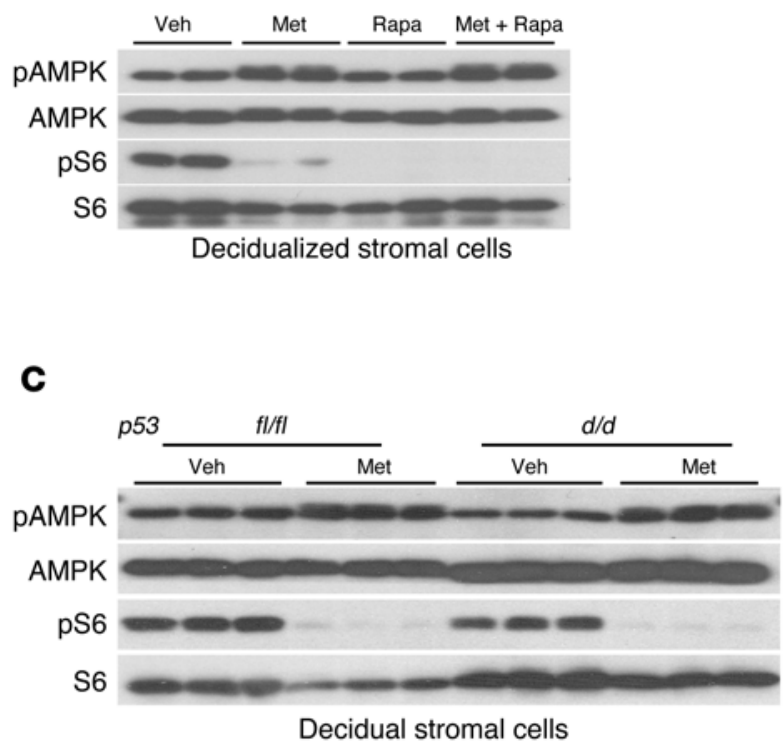

E

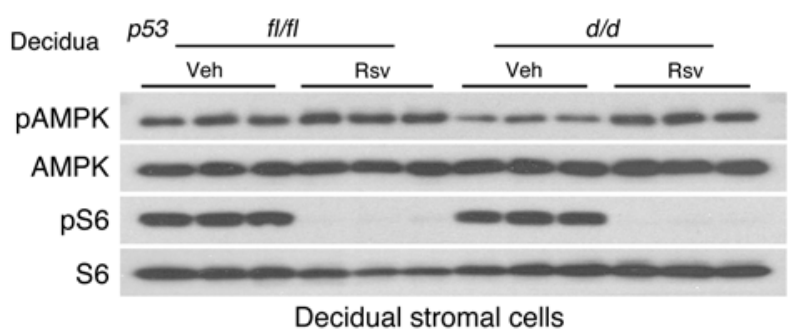

G

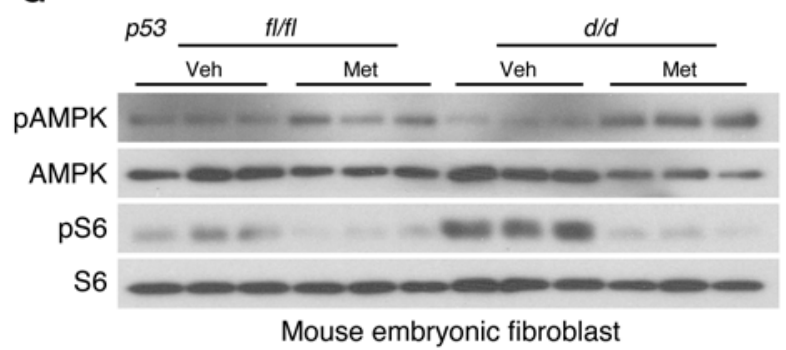

B

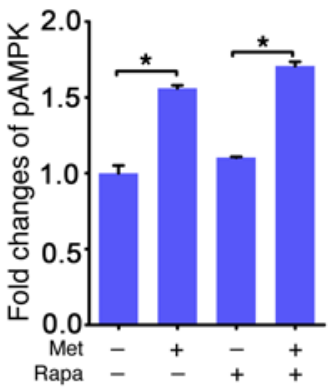

D

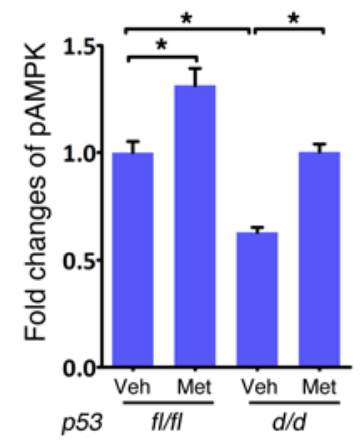

$\mathbf{F}$

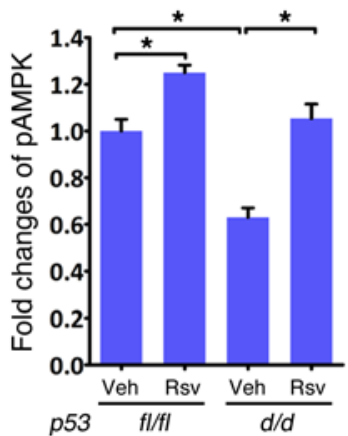

H

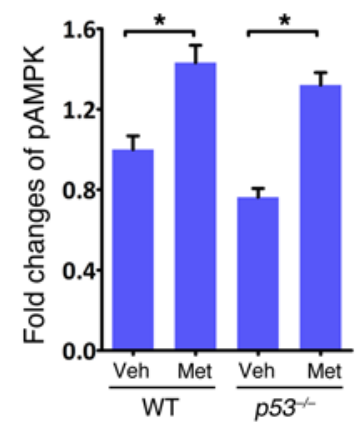

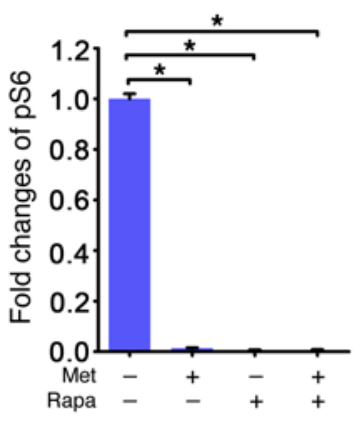
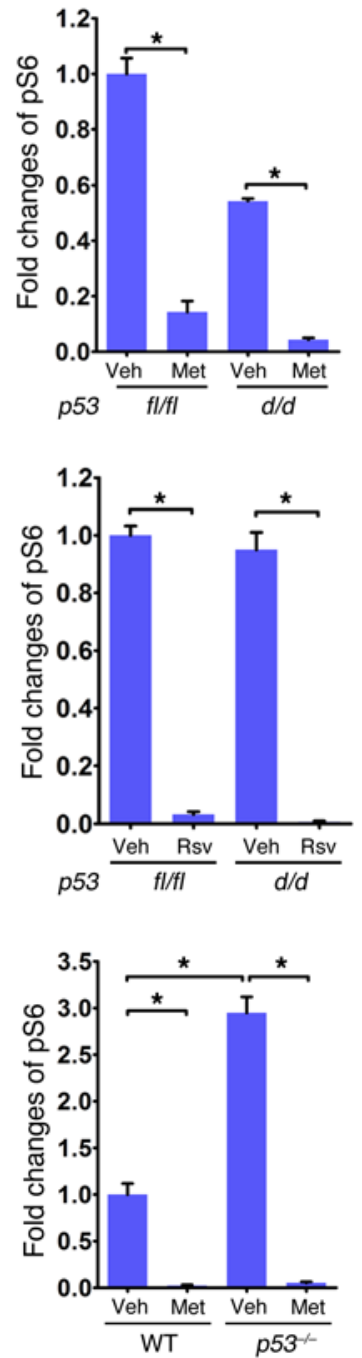

Figure 5. p53 inversely regulates pAMPK and mTORC1 signaling. (A and B) Met or rapamycin (Rapa) inhibited pS6 in decidualized mouse stromal cells; Met alone stimulated pAMPK signaling. (C-F) Met $(10 \mathrm{mM})$ or Rsv $(50 \mu \mathrm{M})$ treatment of $p 53^{f / f l}$ and $p 53^{\mathrm{d} / \mathrm{d}}$ decidualized mouse stromal cells increased pAMPK levels in $p 53^{d / d}$ decidual cells with concomitant decreased pS6 levels. (G and $\mathbf{H}$ ) pS6 levels significantly increased in $p 53^{-/-}$MEFs compared with control cells. pAMPK and pS6 levels in WT and $p 53^{-1-}$ MEFs were inversely regulated by Met. ${ }^{*} P<0.05$, mean \pm SEM, ANOVA.

results, we asked whether Met or Rsv inhibition of mTORC1 activity is dependent on AMPK activity. We exposed Prkaa1 ${ }^{-/-}$Prkaa2 $^{-/-}$ double knockout (referred to here as AMPK dKO) MEFs to Met or Rsv, and, as expected, levels of pAMPK and its target pRAPTOR (28) were not upregulated in AMPK dKO cells. Notably, pS6 levels still showed downregulation in AMPK dKO MEFs (Supplemental Figure 7, A-D), suggesting that Met or Rsv can inhibit mTORC1 signaling through an AMPK-independent pathway, consistent with the results of a recent report (13). Collectively, these results suggest that Met or Rsv can influence mTORC1 signaling through both AMPK-dependent and -independent pathways in a physiological context and cell-dependent manner.

p53-sestrin interactions coordinate AMPK/mTORC1 signaling. To better understand how AMPK and MTORC1 signaling are integrated by $\mathrm{p} 53$, we asked whether sestrins participate in this process. Sestrins are evolutionarily conserved proteins that act as scaffolds 

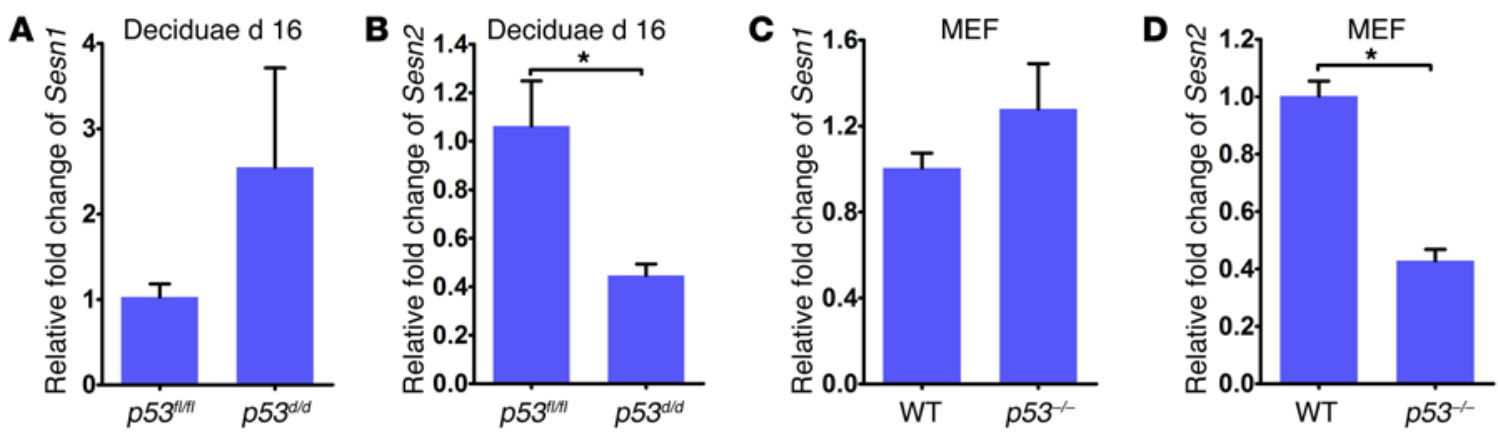

E
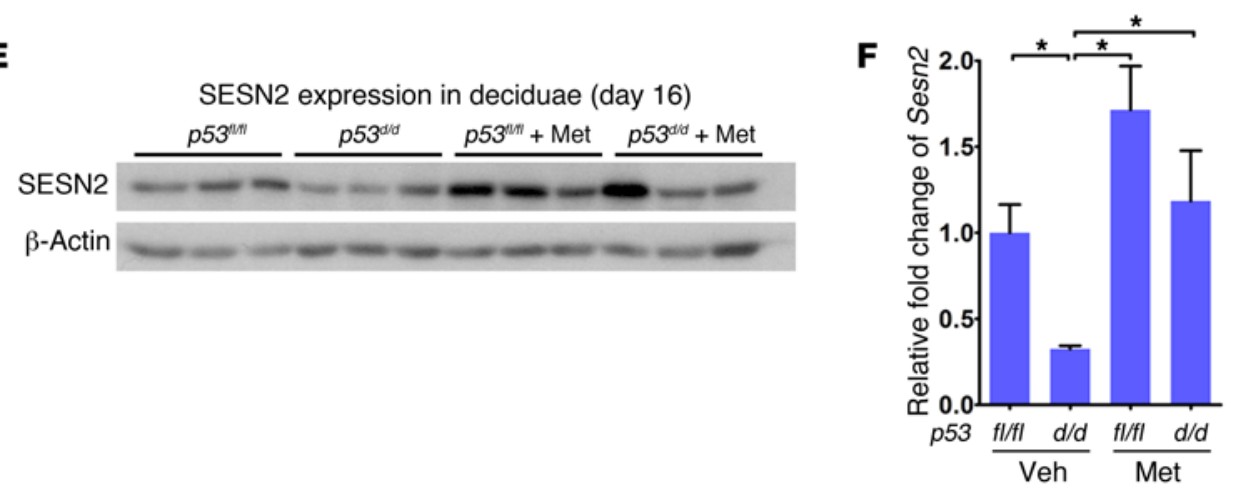

G

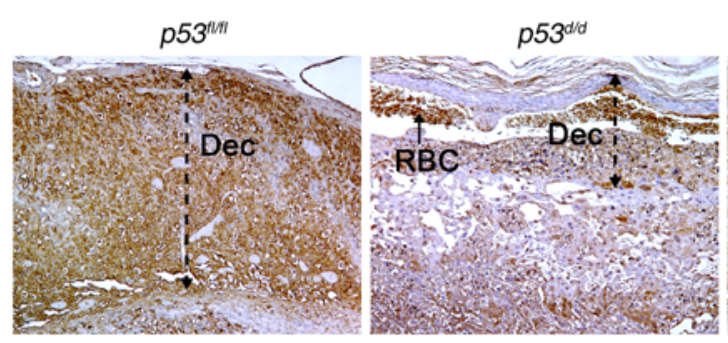

SESN2 localization in deciduae (day 16)

H

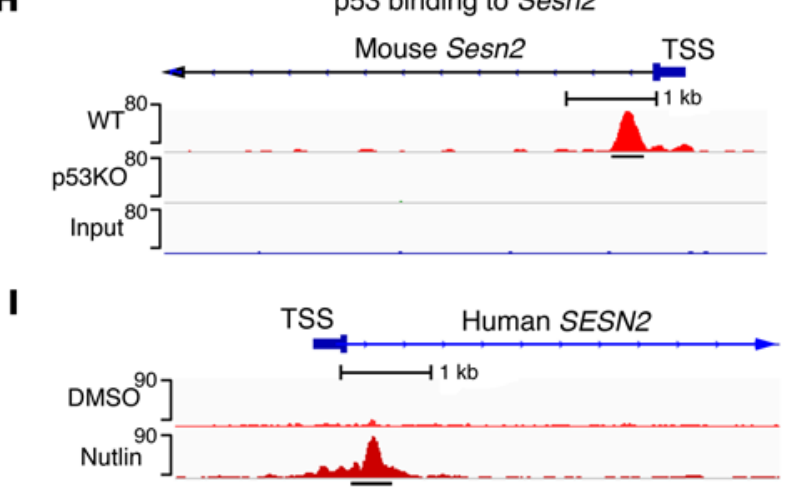

$p 53^{\text {thIt }}+$ Met

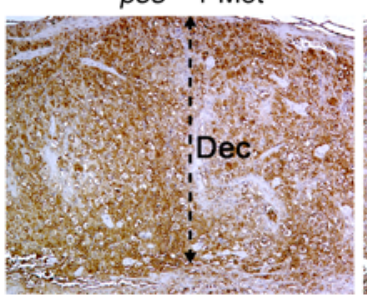

$p 53^{d / d}+$ Met
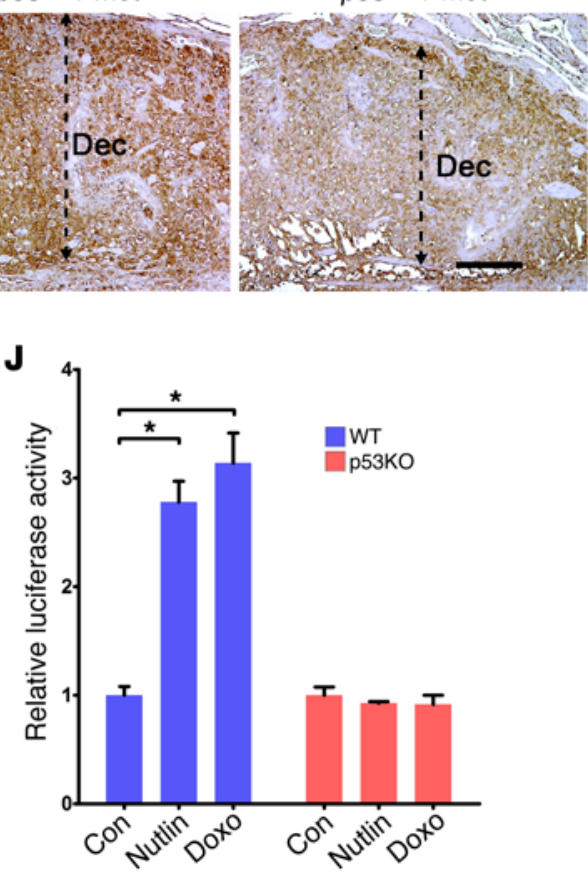

Figure 6. p53 regulates SESN2 expression in deciduae. (A and B) Sesn2 expression is downregulated in $p 53^{d / d}$ deciduae on day 16 with no significant changes in Sesn1 expression compared with $p 53^{f l / f l}$ deciduae ( $n=4,{ }^{*} P<0.05$, mean \pm SEM, Student's $t$ test). (C and D) Sesn2 expression is downregulated in $p 53^{-1-}$ MEFs with no significant changes in Sesn1 expression ( $n=3,{ }^{*} P<0.05$, mean \pm SEM, Student's $t$ test). (E and F) SESN2 protein levels were downregulated in $p 53^{d / d}$ deciduae and upregulated by Met on day 16 ( $n=3,{ }^{*} P<0.05$, mean \pm SEM, ANOVA). (G) SESN2 is downregulated in $p 53^{d / d}$ deciduae, and Met pretreatment upregulated SESN2 expression in $p 53^{d / d}$ deciduae. Dec, decidua; RBC, red blood cells. Scale bar: $250 \mu \mathrm{m}$. (H and I) Putative $\mathrm{p} 53$ binding sites for Sesn2 in mouse and human. (J) Increases in luciferase activity of Sesn2 after treatment with Nutlin or Dox in WT MEFs, but not in p53 ${ }^{-1-}$ MEFs $\left(n=6,{ }^{*} P<0.05\right.$, mean \pm SEM, ANOVA). TSS, transcription start site. 

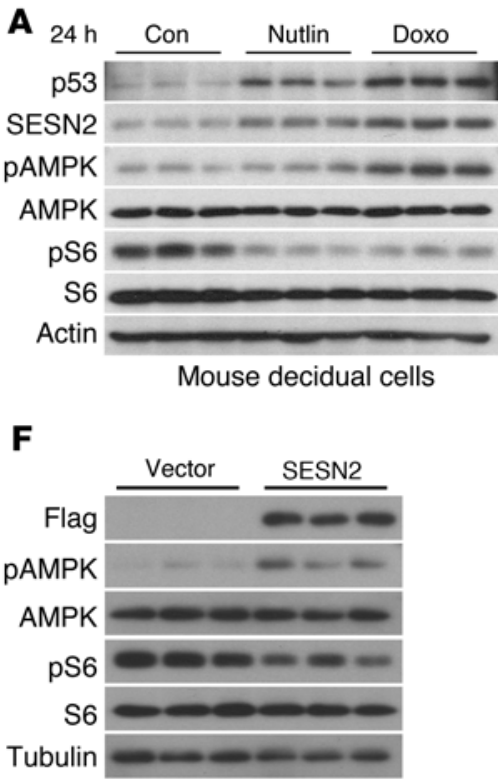
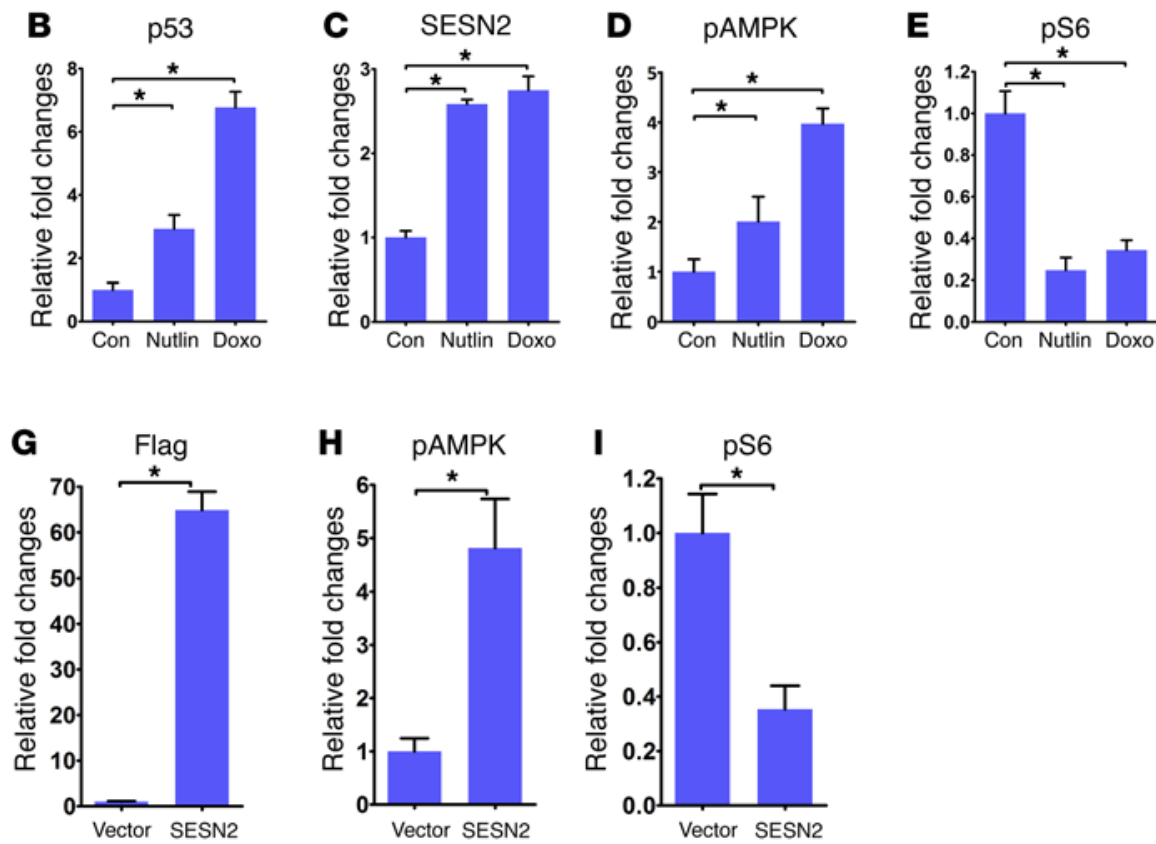

Figure 7. p53 induces SESN2 expression to modulate AMPK and mTORC1 signaling. (A-E) Nutlin and Doxo upregulated the levels of p53, SESN2, and pAMPK with downregulation of pS6 levels in decidualized stromal cells $\left(n=3,{ }^{*} P<0.05\right.$, mean \pm SEM, ANOVA). (F-I) SESN2 overexpression inversely regulated pAMPK and pS6 levels ( $n=3,{ }^{*} P<0.05$, mean \pm SEM, Student's $t$ test).

and are stress-inducible regulators of AMPK/mTORC1 signaling (38). They are thought to attenuate cellular aging by altering cell metabolism and are induced by genotoxic stresses, such as DNA damage responses (20), perhaps through AMPK signaling (21). To address their roles in the decidua in the context of $\mathrm{p} 53$, we first assessed the expression of Sesn 1 and $\operatorname{Sesn} 2$ in $p 53^{f / / l}$ and $p 53^{d / d}$ deciduae on day 16 as well as in WT and $p 53^{-/-}$MEFs. We found that Sesn 2 expression was substantially lower in $p 53^{d / d}$ deciduae compared with floxed deciduae (Figure 6B). Levels of Sesn 2 were also low in $p 53^{-/-}$MEFs (Figure 6D). However, no significant changes in Sesn1 levels were noted in either day 16 deciduae or MEFs (Figure 6 , A and C). Western blotting results also showed reduced SESN2 expression in $p 53^{d / d}$ deciduae on day 16 (Figure 6, E and F). Realtime PCR results showed that Met substantially increased Sesn2 transcript levels in both $p 53^{f / f l}$ and $p 53^{d / d}$ deciduae on day 16 (Supplemental Figure 8). Furthermore, Sesn 2 was found to be primarily localized in deciduae by IHC. We observed that its expression was lower in day $16 p 53^{d / d}$ deciduae, which was substantially improved by Met treatment (Figure 6G). This corroborates other findings that Met or Rsv increases Sesn 2 expression (39-41). We next asked whether p53 can directly regulate the expression of Sesn 2 . Using published Chip-seq data sets (35-37), we found a conserved p53 regulatory element in the first intron of SESN2 in both mice and humans (Figure 6, $\mathrm{H}$ and I). To directly assess the role of p53 in Sesn2 expression, we cloned the p53-enriched fragment into a luciferase reporter construct and transfected it into WT and $p 53^{-1-}$ MEFs. These cells were then treated with Nutlin 3a, a stabilizer of p53 that inhibits the endogenous p53-degrading enzyme MDM2, or doxorubicin (Doxo), a DNA intercalating agent that enhances p53 activity. We found increased luciferase activity in WT MEFs in the presence of Nutlin 3a or Doxo, but not in $p 53^{-1-}$ MEFs (Figure $6 \mathrm{~J})$, suggesting that p53 directly regulates Sesn 2 expression.
To further assess the relationship between p53 and SESN2 and their effects on AMPK and mTORC1 signaling, WT primary stromal cells decidualized in vitro were cultured in the presence or absence of Nutlin 3a or Doxo. We found that both p53 and SESN2 levels were upregulated; these results were associated with increases in pAMPK and attenuation of pS6 levels (Figure 7, A-E), suggesting that SESN2 interacts with p53 to set up a rheostat for reciprocal regulation of pAMPK and pS6 activities. The previous results in cell lines are supportive of our present findings in decidual cells (21). A recent report also shows a relationship among p53, TSC2, and SESN2 to regulate mTORC1 activity through exclusion of lysosomal Rheb (42). Here we now show that overexpression of SESN2 in MEFs upregulates pAMPK levels with concomitant decreases in pS6 levels (Figure 7, F-I). These results provide evidence that AMPK is a downstream target of SESN2 and that increased pAMPK signaling interferes with mTORC1 signaling.

Effects of Met and Rsv are conserved in human decidual cells. We next asked whether activation of AMPK and inhibition of mTORC1 signaling are conserved in human decidual cells. Human uterine fibroblast $(\mathrm{HuF})$ cells were differentiated into decidual cells in culture (43); highly upregulated expression of IGF-binding protein 1 (IGFBP1) and prolactin $(P R L), 2$ reliable decidual markers in humans (43), confirmed successful decidualization (Supplemental Figure 9, A and B). Decidual cells were then treated with Met or Rsv. The results showed a similar signature of reciprocal upregulation of pAMPK with downregulation of pS6 levels by Met (Figure $8, A-D)$. However, Rsv showed limited upregulation of pAMPK but with higher levels of inhibition of pS6. This relationship between pAMPK and pS6 levels was also noted in human primary decidual cells isolated from term placentas when cultured in the presence of Met or Rsv in a dose-responsive manner (Figure 8, E-J). Notably, human decidual tissues and cells cultured in vitro maintained their 
A

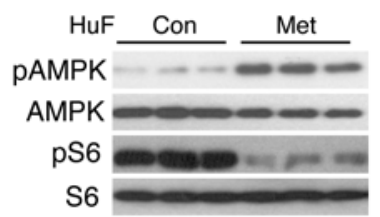

B

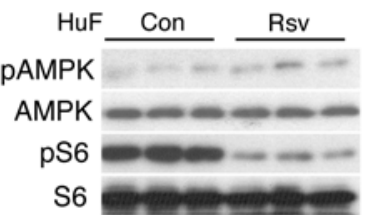

C

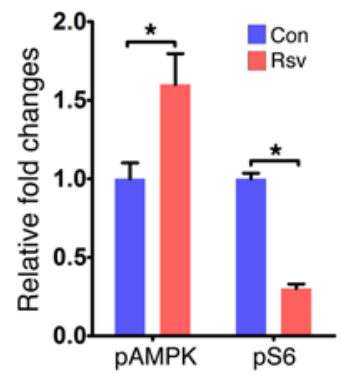

D

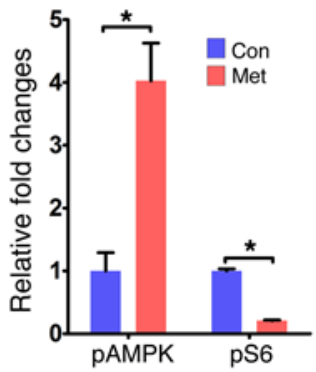

E

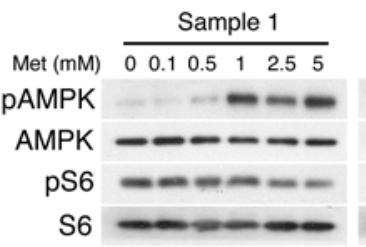

Human decidual cells isolated from term placenta

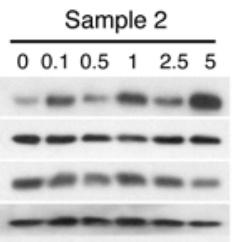

$\frac{\text { Sample } 4}{0.1}$

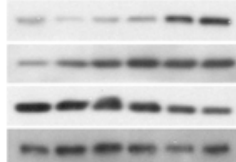

$\mathbf{F}$

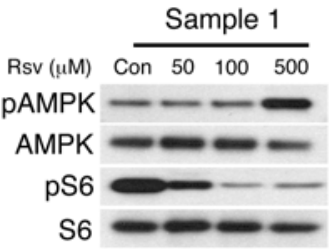

G

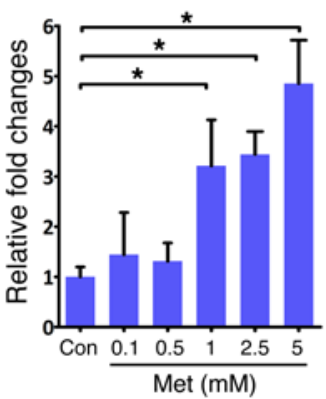

I

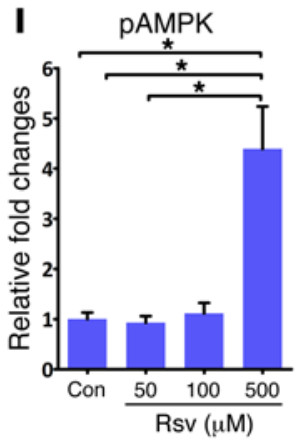

Human decidual cells isolated from term placenta

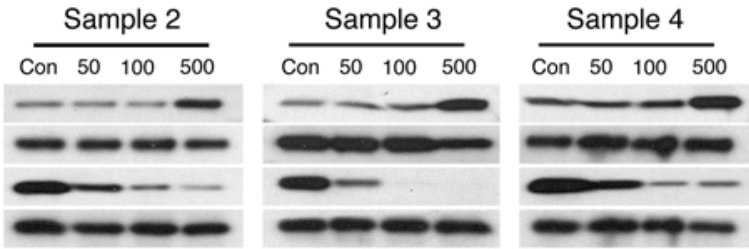

H

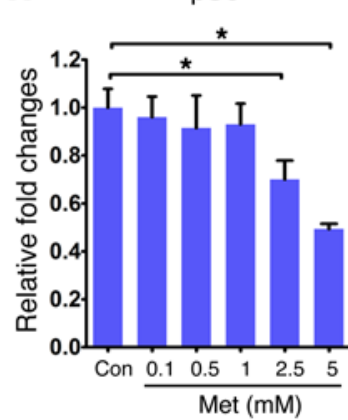

J

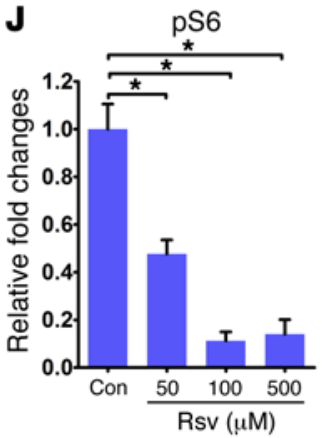

K Human decidual cells

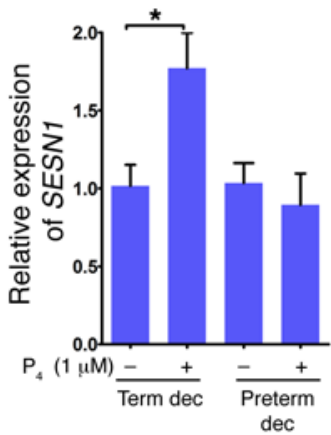

L Human decidual cells

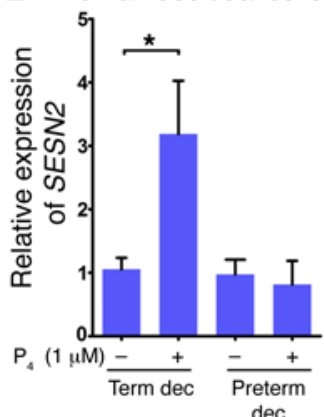

Figure 8. p53-sestrin interactions coordinate AMPK and mTORC1 signaling in human decidual cells. (A-D) Met or Rsv inversely regulated pAMPK and pS6 levels in HuF cells ( $n=3,{ }^{*} P<0.05$, mean \pm SEM, Student's $t$ test). (E-J) pAMPK and pS6 levels were inversely regulated by Met or Rsv in human primary decidual cells isolated from term placentas. Each sample was isolated from a discrete decidua from a single patient. pAMPK and pS6 levels were inversely regulated by Met or Rsv $\left(n=4,{ }^{*} P<0.05\right.$, mean \pm SEM, ANOVA). (K and $\left.\mathbf{L}\right)$ SESN1 and SESN2 mRNA was upregulated by $\mathrm{P}_{4}$ in term decidual cells, but not in preterm decidual cells $\left(n=3,{ }^{*} P<0.05\right.$, mean \pm SEM, Student's $t$ test).

decidual characteristics, expressing decidual cell markers (Supplemental Figure 9, C and D). Additionally, HuF cells decidualized in vitro maintained the expression of decidual markers in the presence or absence of Met or Rsv, suggesting that these compounds do not interfere with the decidualization process (Supplemental Figure 9, $\mathrm{E}-\mathrm{H})$. We also found that pAMPK and mTORC1 signaling shows a positive correlation with the expression of both SESN1 and SESN2 upon exposure to $\mathrm{P}_{4}$ in term decidual cells, while preterm decidual cells were nonresponsive (Figure 8, K and L). Taken together, the results show that this relationship is preserved in human decidual cells and may well be linked to human parturition.

\section{Discussion}

This investigation has identified AMPK as a novel signaling pathway involved in parturition and highlights the role of p53-sestrin interactions that integrate AMPK and mTORC1 signaling to determine parturition timing. We initially used Met and Rsv to rescue PTB syndrome in $p 53^{d / d}$ mice, and the results have been highly instrumental in exploring the molecular linkage of premature decidual senescence with AMPK/mTORC1 signaling in PTB (4). Our study shows that an inverse relationship between AMPK activation and mTORC1 signaling is organized in the decidua by Met or Rsv treatment to restrain premature decidual senescence, 


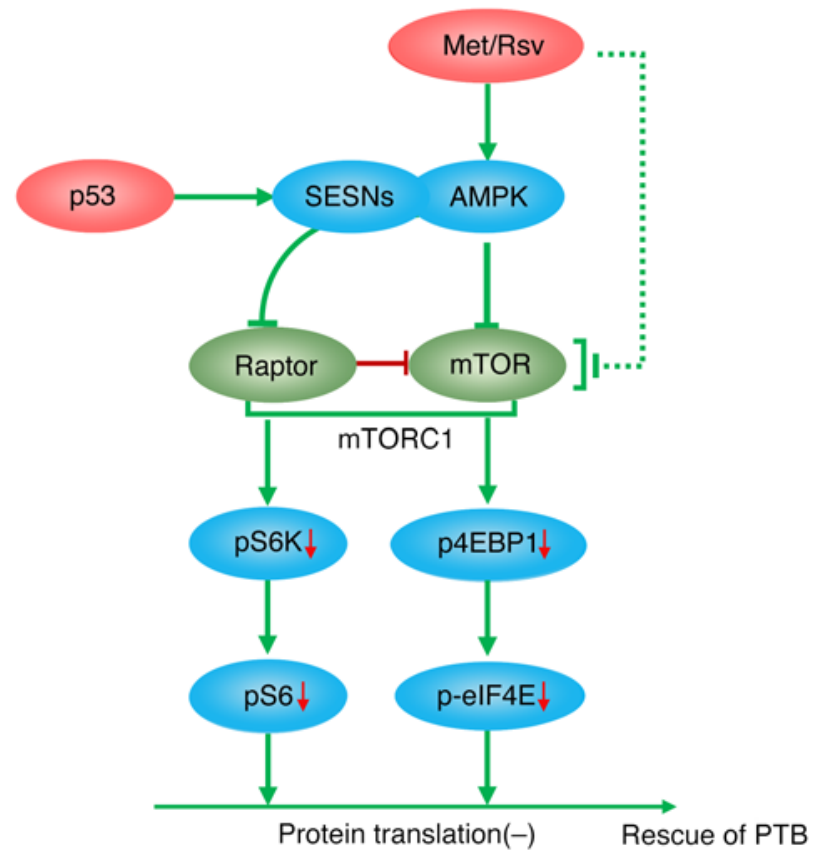

Figure 9. A schematic representation of the role of decidual p53-sestrin interactions to integrate AMPK and mTORC1 signaling in determining parturition timing. Increased AMPK signaling by SESNs in mouse and human decidual cells with downregulation of mTORC1 signaling by $p 53$ and further enhanced by Met or Rsv provides protection against preterm birth (PTB) This downregulation of mTORC1 signaling with increased PAMPK activity is reflected in attenuated protein translation involving pS6K and/or p4EBP1. This perhaps helps rescue PTB.

offering protection against spontaneous and inflammation-exacerbated PTB in $p 53^{d / d}$ mice. The observation that Met and Rsv have protective roles against premature decidual senescence-associated PTB is an exciting development since both are now known to have anti-aging properties and reportedly extend lifespan $(17,44)$. Met can inhibit mTORC1 activity via AMPK-dependent or -independent pathways (7), whereas Rsv inhibits the hyperactivated mTORC1 pathway (45). Although mTORC1 signaling has taken center stage in cellular senescence, our in vivo and in vitro results suggest that a tightly regulated reciprocal integration of AMPK and mTORC1 signaling together plays a significant role in the decidual senescence in both mice and humans.

Attenuation of cellular senescence, evident from reduced SA- $\beta$-gal staining, sparse numbers of $\gamma \mathrm{H} 2 \mathrm{AX}$-positive cells, and improved markers of decidual health with Met pretreatment, is a testament to the beneficial effects of this drug during pregnancy. Our observations of the reciprocal relationship between pAMPK and $\mathrm{mTORC} 1 \mathrm{in}$ response to Met or Rsv, preserved in both deciduae in vivo and primary stromal cells decidualized in culture, as well as in primary human cultured decidual cells, present the importance of the reciprocal interactions between these 2 pathways in parturition. Our observation of Met's effectiveness to upregulate pAMPK levels in decidual cells in the face of inhibition of MTORC1 activity by rapamycin suggests that Met's influence on AMPK signaling need not necessarily be limited by rapamycin. However, downregulation of pS6 levels in AMPK dKO MEFs by Met or Rsv indicates that they can act independently of AMPK to attenuate mTORC1 activity. These observations suggest that the relationship between AMPK and mTORC1 signaling is complex and context dependent, which corroborates previous findings in other systems $(13,46)$. The observation of the requirement of a low dose of Met $(1 \mathrm{mg} / \mathrm{kg} \mathrm{BW}$ per mouse) to be effective in vivo as opposed to a much higher concentration $(10 \mathrm{mM})$ in cultured decidual cells was perhaps due to higher expression of $O c t 1$ and $O c t 3$ in tissues in vivo as compared with much lower expression in cultured cells; OCTs serve as transporters to deliver Met inside the cell. In this respect, several studies used Met at $10 \mathrm{mM}$ to be effective $(13,47,48)$.

Our present results also show a relationship between p53-sestrin and AMPK/mTORC1 signaling and their integration to optimize decidual function. Sestrins are known transcriptional targets of p53 with antioxidant and anti-aging properties (20, 21, 49). Deletion of sestrins incurs abnormal accumulation of metabolically active oxidants and hastens cellular aging (50). There is evidence that SESN2 can also target mTORC1 signaling via an AMPK-independent mechanism by directly binding with Raptor, a component of the mTORC1 complex, or with GATOR2 in an amino acid-sensitive manner, or with RAG A/B-RAG C/D GTPases to promote mTORC1 translocation to lysosome (21, 51-53). Our observation of transcriptional activation of SESN2 by $p 53$, its low levels in $p 53^{d / d}$ decidual cells, and its unresponsiveness to Nutlin $3 \mathrm{a}$ or Doxo in $p 53^{-1-}$ MEFs provide evidence that dysregulation of sestrins is relevant to premature decidual senescence and PTB in $p 53^{d / d}$ dams. The induction of p53 by Nutlin 3a or Doxo along with increases in SESN2 and pAMPK levels concomitantly with downregulation of pS6 levels in decidual cells suggests that p53-SESN2 interactions can influence AMPK/mTORC1 signaling in deciduae to coordinate parturition timing. Our findings of stimulation of SESN1 and SESN2 by $\mathrm{P}_{4}$ in term decidual cells, but its inability to influence their expressions in human preterm decidual cells, suggest that this complex system also operates in calibrating human parturition timing.

The robust protective effects of Met or Rsv against PTB in $p 53^{d / d}$ females are clinically relevant. If used cautiously, these drugs can safely target decidual senescence by establishing reciprocal interactions between AMPK and mTORC1 signaling. We can predict Met's safety in pregnancy through its approval for the treatment of type 2 diabetes and its frequent use with clomiphene as a first line of therapy for ovulation induction in the treatment of infertile patients with polycystic ovary syndrome (54). A metaanalysis of 8 small studies has shown that it does not yield major adverse effects, including maternal hyperinsulinemia and fetal hypoglycemia, if consumed in the first trimester (10).

Rsv is an increasingly studied agent for its antioxidant and anticancer properties (55). It is reported to activate AMPK to ameliorate aging (17, 56-60). In mice, long-term administration of Rsv has been shown to delay aging-related deterioration and shows gene expression patterns similar to those induced by caloric restriction (61). In a mouse study, Rsv increased uterine arterial blood flow velocity and fetal weight in $\mathrm{eNos}^{-/}$and $\mathrm{Comt}^{-/-}$mice, suggesting potential as a therapeutic strategy for preeclampsia and fetal growth restriction (22). There is now evidence that an inflammatory stimulus insufficient to induce PTB during the late gestational stage is likely to trigger neurological disorders in the newborn later in life $(62,63)$. It would be interesting to see wheth- 
er Met or Rsv could alleviate these risks in future investigation. However, both risks and benefits must be carefully weighed with regard to use of Met or Rsv during pregnancy.

In essence, this study has gleaned that PTB in genetically predisposed mice can be attenuated with intermittent administration of low doses of Met, and that both Met and Rsv attenuate decidual mTORC1 activity by increasing AMPK signaling to improve decidual health. While studying the effect of these drugs, we generated valuable insight regarding transcriptional regulation of sestrins by p53 to fine-tune and integrate AMPK and mTORC1 signaling pathways in decidual senescence and parturition timing (Figure 9). This study's clinical value is apparent since human decidual cells showed a similar signature of increased AMPK signaling with reduced mTORC1 activity upon exposure to Met or Rsv treatment. These preclinical studies could provoke interest in designing a stepwise approach to strategies targeting human PTB.

\section{Methods}

Mice and drug treatment. Trp5 $3^{\text {loxP/loxp }} \mathrm{PgrCre} /+$ mice were maintained by mating of $\operatorname{Trp} 53^{\text {loxP/loxP }}$ mice (FVB/129) with $\mathrm{Pgr}{ }^{\mathrm{Cre} /+}$ mice (C57BL/6-129) to generate mice with uterine deletion of $\operatorname{Tr} p 53\left(p 53^{d / d}\right.$ mice). $\operatorname{Tr} p 53^{\text {loxP/loxP }}$ mice were obtained from the Mouse Models of Human Cancers Consortium, while $\mathrm{Pgr}{ }^{\mathrm{Cr} /+}$ mice were initially provided by John Lydon and Francesco DeMayo (Baylor College of Medicine, Houston, TX). Ultrapure TLR4-specific LPS (10 $\mu \mathrm{g} / \mathrm{mouse}$, i.p.; InvivoGen) was administered on day 16 of pregnancy at 1200 hours. Met was suspended in $5 \%$ PEG400 and 5\% Tween-80 dissolved in water and was given by an oral gavage as indicated ( $1 \mathrm{mg} / \mathrm{kg}$ BW per dose) on days 8,10 , and 12. Rsv was suspended in $0.5 \%$ methylcellulose dissolved in water and given by oral gavage as indicated ( $30 \mathrm{mg} / \mathrm{kg}$ BW per dose) on days 8 , 10,12 , and 14 . The control group received vehicle alone. Progesterone $\left(\mathrm{P}_{4}\right)$ was dissolved in sesame oil and administered s.c. (1 or $2 \mathrm{mg} /$ mouse) 4 hours before LPS injection (10 $\mu \mathrm{g} /$ mouse) .

Analysis of parturition. Parturition events were monitored as described previously (4). Briefly, parturition was checked from day 16 through day 21 by observation of mice daily, morning (0600-0700 hours), noon (1200 hours), and evening (1800-2000 hours). Birth timing was defined by the observation of the first born pup. PTB was defined as birth occurring earlier than day 19 of pregnancy (day $1=$ vaginal plug in the morning). Dystocia was defined as difficult delivery lasting more than 12 hours. Resorption sites and placental scars were identified in dams showing preterm or difficult deliveries by examination of the uterus. The number of pups/masses delivered was compared with the number of resorption sites and placental scars.

Measurement of PG profiles. Implantation sites from which fetuses and placentas had been removed were collected on day 17 of pregnancy. These tissues were flash-frozen and stored at $-80^{\circ} \mathrm{C}$ until used for extractions. For intracellular measurement of PGs, stromal cells from $p 53^{f / f l}$ and $p 53^{d / d}$ mice were decidualized with hormones for 4 days in culture and then treated with Met and Rsv for 24 hours and 6 hours, respectively. These cells were washed with cold PBS and pelleted. Pellets were stored frozen at $-80^{\circ} \mathrm{C}$. Methanolic extracts were partially purified using C18 solid-phase extraction columns (Agilent), and PGs were quantified by HPLC-tandem mass spectrometry as previously described (4).

Cell culture and treatments. Stromal cells from day 4 of pregnancy were collected by enzymatic digestion as described previously (29). The cells were cultured overnight before the initiation of decidualiza- tion by treatment of estradiol- $17 \beta\left(\mathrm{E}_{2}, 10 \mathrm{nM}\right)$ and progesterone $\left(\mathrm{P}_{4}\right.$, $1 \mu \mathrm{M})$ in phenol red-free DMEM/F12 media supplemented with charcoal-stripped 1\% FBS (vol/vol). These cells were treated with Met $(10 \mathrm{mM})$, Rsv $(50 \mu \mathrm{M})$, AICAR $(500 \mu \mathrm{M})$, rapamycin $(1 \mathrm{nM})$, Nutlin 3a $(5 \mu \mathrm{M})$, or doxorubicin $(0.5 \mu \mathrm{M})$.

WT and $p 53^{-/-}$mouse embryonic fibroblasts (MEFs) were generated in house, and MEFs from Prkaa1-/-Prkaa2-/- (AMPK dKO) mice (generated by the Viollet laboratory, Université Paris Descartes) were cultured in DMEM media supplemented with $10 \% \mathrm{FBS}$ (vol/vol). Human uterine fibroblast (HuF) cells, generated and shared by the late Stuart Handwerger (Cincinnati Children's Hospital Medical Center), were cultured in DMEM/F12 containing 5\% charcoal-stripped FBS (vol/vol) and decidualized with $\mathrm{E}_{2}(10 \mathrm{nM})$, medroxyprogesterone acetate $(1 \mu \mathrm{M})$, and $\mathrm{PGE}_{2}$ $(10 \mathrm{nM})$ in DMEM/F12 containing 1\% charcoal-stripped FBS (vol/vol) for 6 days. The medium was changed every other day (43).

In situ hybridization. In situ hybridization was performed as described previously (4). Implantation sites were collected and flash-frozen. Cryosections $(12 \mu \mathrm{m})$ were mounted onto poly-L-lysinecoated slides, fixed in cold $4 \%$ paraformaldehyde, acetylated, and hybridized at $45^{\circ} \mathrm{C}$ for 4 hours in formamide hybridization buffer containing ${ }^{35}$ S-labeled Prlr (long isoform) and Ptgs2 (Cox2) cRNA probes. RNase A-resistant hybrids were detected by autoradiography using Kodak NTB-2 liquid emulsion. Both $p 53^{f / f l}$ and $p 53^{d / d}$ sections were placed onto the same slide and processed for hybridization.

IHC and IF staining. Immunostaining was performed in formalin-fixed, paraffin-embedded sections using specific antibodies to $\gamma \mathrm{H} 2 \mathrm{AX}$ and SESN2. Sections from $p 53^{f / / f l}, p 53^{d / d}$, or Met-treated tissues on day 16 of pregnancy were processed onto the same slide. For IF studies, paraformaldehyde-fixed frozen sections $(12 \mu \mathrm{m})$ were blocked with $5 \%$ BSA in PBS and immunostained by antibodies for pAMPK and PECAM. Signals were visualized by secondary antibody conjugated with Cy3 fluorophore (Jackson ImmunoResearch Laboratories). Sections were counterstained with Hoechst 33342 ( $2 \mu \mathrm{g} / \mathrm{ml}$; Life Technologies). Applications and descriptions of antibodies are presented in Supplemental Table 1.

$S A$ - $\beta$-gal staining. Staining of SA- $\beta$-gal activity was performed as described previously (4). Frozen sections were fixed in $0.5 \%$ glutaraldehyde in PBS and stained for 6 hours in PBS (pH 5.5) containing $1 \mathrm{mM} \mathrm{MgCl}, 1 \mathrm{mg} / \mathrm{ml} \mathrm{X}$-gal, and $5 \mathrm{mM}$ each of potassium ferricyanide and potassium ferrocyanide. Sections were counterstained with eosin.

Western blotting. Protein extraction and Western blotting were performed as previously described (4). Antibodies to pRAPTOR, RAPTOR, pS6, S6, pS6K, S6K, pAMPK, AMPK, p4EBP1, 4EBP1, p-eIF4E, eIF4E, SESN2, p53, Actin, and $\alpha$-Tubulin were used (Supplemental Table 1). Bands were visualized using an ECL Prime Western blotting detection system (GE Healthcare). Actin and $\alpha$-tubulin served as loading controls.

RNA isolation and quantitative PCR. RNA was prepared from homogenized tissues using TRIzol reagent (Invitrogen). RNA extraction was performed as described previously (4). Quantitative PCR was performed using StepOnePlus Real-Time PCR System (Applied Biosciences). PCR was performed using the following primers: $5^{\prime}$-GCAGATGTACCGCACTGAGATTC-3' and 5'-ACCTTTGGGCTTACTCCATTGATA-3' for mouse Rpl7; 5'-CCAGATGTGGGACAGTTA-3' and 5'-CCAGATGTGGGACAGTTA-3' for Sesn1; 5'-ACTGCGTCTTTGGCATCA-3' and 5'-CATCCTACGGGTCGTCTTCT-3' for Sesn2; 5'-TTCAGGGAGAAGCGTTTGC-3' and 5'-ACACACTCTATCACTGGCACC-3' for Ptgs2 (encoding COX2); 5'-GGATGTGACTTACATTGTTGAACCA-3' and 5'-TACCCACAGATATGTTTTTTTGTCTTT-3' for Prlr; 5'-CCAAACT- 
GCAACAAGAATG-3' and 5'-GTAGACGCACCAGCAGAG-3' for human IGFBP1; 5'-AAGCTGTAGAGATTGAGGAGCAAA-3' and 5'-TCAGGATGAACCTGGCTGACTA-3' for human PRL; 5'-GATTGCCCGACTCCCTTGG-3' and 5'-GTCTAGCCAGAGTTTCACCGT-3' for human PTGS2 (encoding COX2); 5'-GGGAGACAGCATTGGAAAACAT-3' and 5'-TGGAGGATCTCCTTTTCTGGGA-3' for human SESN1; 5'-GCAGGGCCATCTGGAACTAT-3' and 5'-AGTTCACGTGGACCTTCTCTG-3' for human SESN2; 5'-TCGGCCTTAGAACCCCAGTA-3' and 5'-GAGATCCCTCCGCAGAATCG-3' for human UBC. Rpl7 and UBC served as housekeeping genes for mouse and human, respectively.

Mouse Sesn 2 overexpression. Mouse Sesn 2 cDNA was amplified and inserted into pCMV-tag2B containing a Flag tag. This plasmid was transfected in MEFs by Lipofectamine 2000. Flag antibody was used to confirm the expression of Sesn2. The primers used for cDNA amplification were 5'-ATCGTAGCGGACTCCGAGTGCCATT- 3 ' and 5'-GGTCATGTAGCGGGTGATGGCGCGC-3'.

Measurement of serum $P_{4}$ levels. Mouse blood samples were collected on day 16 of pregnancy at the prescribed time after treatments. Serum levels of $\mathrm{P}_{4}$ were measured by enzyme immunoassay kits (Cayman Chemical).

Isolation and culture of human decidual cells. Term and preterm human decidual cells were isolated and cultured according to previously described protocols (4). Briefly, deciduae were scraped from the maternal surface of the chorion and used for RNA extraction. For cell culture, the deciduae were minced, and digested in Ham's F-10 media containing $25 \mathrm{mg} / \mathrm{ml}$ collagenase and $6.25 \mathrm{U} / \mathrm{ml}$ DNase in a shaking water bath at $37^{\circ} \mathrm{C}$ for 30 minutes. Digested samples were passed through a 23-gauge needle to dissociate remaining cell clusters, centrifuged at $250 \mathrm{~g}$ for 5 minutes, and washed in the culture media. The cell pellet was resuspended in 20\% Percoll, layered on a discontinuous Percoll gradient (60\%:50\%:40\%), and centrifuged at $540 \mathrm{~g}$ for 20 minutes. The upper cell layer was collected, washed, centrifuged, resuspended in $40 \%$ Percoll, layered on a discontinuous Percoll gradient (55\%:50\%:45\%), and centrifuged at $540 \mathrm{~g}$ for 20 minutes. The upper cell layer was washed and resuspended in DMEM/F-12 media containing $5 \%$ FBS. Cells were passaged at least 3 times and plated in 12 -well plates at $2 \times 10^{5}$ cells per well for experiments.

Identification of whole-genome p53 binding sites, construction of a luciferase reporter, and transfection. The databases used in this study were from GEO GSE46240 (36), GSE26360 (35), and GSE58740 (37). Sequenced reads were aligned to the mouse genome $(\mathrm{mm} 9)$ or human genome (hg19) by bowtie, and reads with more than 2 aligned positions were removed. Only nonredundant reads that passed the quality score were kept for downstream analysis. ChIP-seq tags were then analyzed using MACS2 (Model-Based Analysis of ChIP-Seq) to identify the peaks. Mouse Sesn2 intron localized in chr4:132509504-132509997 was inserted in pGL3-Basic and transfected in MEFs with pRL-TK by Lipofectamine 2000. These cells were treated with Nutlin or doxorubicin for 6 hours and then collected for luciferase measurement.
Statistics. Statistical analyses were performed using 2-tailed Student's $t$ test, ANOVA, and $\chi^{2}$ tests. $P$ values less than 0.05 were considered statistically significant.

Study approval. All mice used in this investigation were housed in the Cincinnati Children's Hospital Medical Center Animal Care Facility according to NIH and institutional guidelines for the use of laboratory animals. All protocols of the present study were reviewed and approved by the Cincinnati Children's Hospital Research Foundation IACUC. Mice were given autoclaved rodent LabDiet 5010 (Purina) and UV light-sterilized reverse osmosis/deionization (RO/DI) constant-circulation water ad libitum and were housed under a constant 12-hour light/12-hour dark cycle.

For human samples, term and preterm human placentas were obtained from women with singleton vaginal term or preterm delivery. None of the women undergoing term vaginal delivery showed any clinical or pathological signs of preterm delivery, infection, or other maternal or placental diseases. Three women all with singleton preterm vaginal delivery were between 27 and 37 years old. One, a primipara, had PTB at 23 weeks and 4 days; the second, also a primipara with a past history of PTB, gave PTB at 23 weeks and 5 days; and the third had premature rupture of membranes and gave PTB at 31 weeks. They did not show clinical or pathological signs of other maternal or placental diseases apart from preterm delivery. Newborns did not have any apparent birth or chromosomal abnormalities. Collection and processing of human placental samples were approved by the research ethics committee at the University of Tokyo under the approved IRB protocol no. 3456, and all patients provided written informed consent.

\section{Author contributions}

All contributing authors agreed to submission of the manuscript for publication. WD, JC, YH, HBB, and SKD conceived and designed the study. WD, JC, JY, AB, HH, and EL performed experiments. $\mathrm{BV}$ and $\mathrm{YH}$ provided critical tools and reagents and reviewed the results. WD, JC, BV, HBB, YH, and SKD wrote the manuscript.

\section{Acknowledgments}

This work was supported in part by grants from the March of Dimes (21-FY12-127 and 22-FY13-543) and the NIH (HD068524 and DA06668) to SKD. JC was a National Research Service Awardee (NIA/NIH F30 AG040858). We thank Katie A. Gerhardt for her excellent editing of the manuscript.

Address correspondence to: Sudhansu K. Dey, Division of Reproductive Sciences, Cincinnati Children's Hospital Medical Center, 3333 Burnet Avenue, Cincinnati, Ohio 45229-3039, USA. Phone: 513.803.1158; E-mail: sk.dey@cchmc.org.

J. Cha's present address is: Department of Medicine, Vanderbilt University Medical Center, Nashville, Tennessee, USA.
1. Goldenberg RL, Culhane JF, Iams JD, Romero R. Epidemiology and causes of preterm birth. Lancet. 2008;371(9606):75-84.

2. Romero R, Dey SK, Fisher SJ. Preterm labor: one syndrome, many causes. Science. 2014;345(6198):760-765.

3. Elovitz MA, Mrinalini C. Animal models of preterm birth. Trends Endocrinol Metab. 2004;15(10):479-487.

4. Cha J, et al. Combinatory approaches prevent preterm birth profoundly exacerbated by gene-environment interactions. JClin Invest. 2013;123(9):4063-4075.

5. Hirota Y, Daikoku T, Tranguch S, Xie H, Brad- shaw HB, Dey SK. Uterine-specific p53 deficiency confers premature uterine senescence and promotes preterm birth in mice. J Clin Invest. 2010;120(3):803-815

6. Hirota Y, Cha J, Yoshie M, Daikoku T, Dey SK. Heightened uterine mammalian target of rapamycin complex 1 (mTORC1) signaling provokes 
preterm birth in mice. Proc Natl Acad Sci US A. 2011;108(44):18073-18078.

7. Sivalingam VN, Myers J, Nicholas S, Balen AH, Crosbie EJ. Metformin in reproductive health, pregnancy and gynaecological cancer: established and emerging indications. Hum Reprod Update. 2014;20(6):853-868.

8. Sander C, Madsen KD, Hyrup B, Nielsen HM, Rantanen J, Jacobsen J. Characterization of spray dried bioadhesive metformin microparticles for oromucosal administration. Eur J Pharm Biopharm. 2013;85(3 pt A):682-688.

9. National Institute for Health and Clinical Excellence. NICE Web site. https://www.nice.org.uk/. Accessed June 29, 2016.

10. Gilbert C, Valois M, Koren G. Pregnancy outcome after first-trimester exposure to metformin: a meta-analysis. Fertil Steril. 2006;86(3):658-663.

11. Foretz M, Guigas B, Bertrand L, Pollak M, Viollet B. Metformin: from mechanisms of action to therapies. Cell Metab. 2014;20(6):953-966.

12. Wheaton WW, et al. Metformin inhibits mitochondrial complex I of cancer cells to reduce tumorigenesis. Elife. 2014;3:e02242.

13. Kalender A, et al. Metformin, independent of AMPK, inhibits mTORC1 in a rag GTPase-dependent manner. Cell Metab. 2010;11(5):390-401.

14. Anisimov VN, et al. Metformin slows down aging and extends life span of female SHR mice. Cell Cycle. 2008;7(17):2769-2773.

15. Check Hayden E. Anti-ageing pill pushed as bona fide drug. Nature. 2015;522(7556):265-266.

16. Lagouge $\mathrm{M}$, et al. Resveratrol improves mitochondrial function and protects against metabolic disease by activating SIRT1 and PGC-1alpha. Cell. 2006;127(6):1109-1122

17. Park SJ, et al. Resveratrol ameliorates agingrelated metabolic phenotypes by inhibiting cAMP phosphodiesterases. Cell. 2012;148(3):421-433.

18. Bitterman JL, Chung JH. Metabolic effects of resveratrol: addressing the controversies. Cell Mol Life Sci. 2015;72(8):1473-1488.

19. Budanov AV, et al. Identification of a novel stress-responsive gene Hi95 involved in regulation of cell viability. Oncogene. 2002;21(39):6017-6031.

20. Budanov AV, Sablina AA, Feinstein E, Koonin EV, Chumakov PM. Regeneration of peroxiredoxins by p53-regulated sestrins, homologs of bacterial AhpD. Science. 2004;304(5670):596-600.

21. Budanov AV, Karin M. p53 target genes sestrin1 and sestrin 2 connect genotoxic stress and $\mathrm{mTOR}$ signaling. Cell. 2008;134(3):451-460.

22. Poudel R, et al. Effects of resveratrol in pregnancy using murine models with reduced blood supply to the uterus. PLoS One. 2013;8(5):e64401.

23. Kim YH, Kim YS, Roh GS, Choi WS, Cho GJ. Resveratrol blocks diabetes-induced early vascular lesions and vascular endothelial growth factor induction in mouse retinas. Acta Ophthalmol. 2012;90(1):e31-e37.

24. Duca FA, et al. Metformin activates a duodenal Ampk-dependent pathway to lower hepatic glucose production in rats. Nat Med. 2015;21(5):506-511.

25. Sedelnikova OA, Horikawa I, Zimonjic DB, Popescu NC, Bonner WM, Barrett JC. Senescing human cells and ageing mice accumulate DNA lesions with unrepairable double-strand breaks.
Nat Cell Biol. 2004;6(2):168-170.

26. Foretz M, et al. Metformin inhibits hepatic gluconeogenesis in mice independently of the LKB1/ AMPK pathway via a decrease in hepatic energy state. J Clin Invest. 2010;120(7):2355-2369.

27. Price NL, et al. SIRT1 is required for AMPK activation and the beneficial effects of resveratrol on mitochondrial function. Cell Metab. 2012;15(5):675-690.

28. Gwinn DM, et al. AMPK phosphorylation of raptor mediates a metabolic checkpoint. Mol Cell. 2008;30(2):214-226.

29. Ma X, et al. Decidual cell polyploidization necessitates mitochondrial activity. PLoS One. 2011;6(10):e26774.

30. Alam SM, et al. A uterine decidual cell cytokine ensures pregnancy-dependent adaptations to a physiological stressor. Development. 2007;134(2):407-415.

31. He L, Wondisford FE. Metformin action: concentrations matter. Cell Metab. 2015;21(2):159-162.

32. Ma XM, Blenis J. Molecular mechanisms of mTOR-mediated translational control. Nat Rev Mol Cell Biol. 2009;10(5):307-318.

33. Inoki K, Zhu T, Guan KL. TSC2 mediates cellular energy response to control cell growth and survival. Cell. 2003;115(5):577-590.

34. Shackelford DB, Shaw RJ. The LKB1-AMPK pathway: metabolism and growth control in tumour suppression. Nat Rev Cancer. 2009;9(8):563-575.

35. Li M, He Y, Dubois W, Wu X, Shi J, Huang J. Distinct regulatory mechanisms and functions for p53-activated and p53-repressed DNA damage response genes in embryonic stem cells. Mol Cell. 2012;46(1):30-42.

36. Kenzelmann Broz D, et al. Global genomic profiling reveals an extensive p53-regulated autophagy program contributing to key $\mathrm{p} 53$ responses. Genes Dev. 2013;27(9):1016-1031.

37. Sammons MA, Zhu J, Drake AM, Berger SL. TP53 engagement with the genome occurs in distinct local chromatin environments via pioneer factor activity. Genome Res. 2015;25(2):179-188.

38. Budanov AV, Lee JH, Karin M. Stressin' Sestrins take an aging fight. $E M B O \mathrm{Mol} \mathrm{Med}$. 2010;2(10):388-400.

39. Ben-Sahra I, et al. Sestrin2 integrates Akt and mTOR signaling to protect cells against energetic stress-induced death. Cell Death Differ. 2013;20(4):611-619.

40. Jin SH, et al. Resveratrol inhibits LXR $\alpha$-dependent hepatic lipogenesis through novel antioxidant Sestrin2 gene induction. Toxicol Appl Pharmacol. 2013;271(1):95-105.

41. Seo K, Seo S, Han JY, Ki SH, Shin SM. Resveratrol attenuates methylglyoxal-induced mitochondrial dysfunction and apoptosis by Sestrin 2 induction. Toxicol Appl Pharmacol. 2014;280(2):314-322.

42. Agarwal S, Bell CM, Taylor SM, Moran RG. p53 deletion or hotspot mutations enhance mTORC1 activity by altering lysosomal dynamics of TSC2 and Rheb. Mol Cancer Res. 2016;14(1):66-77.

43. Schroeder JK, Kessler CA, Handwerger S. Critical role for TWIST1 in the induction of human uterine decidualization. Endocrinology. 2011;152(11):4368-4376.

44. Martin-Montalvo A, et al. Metformin improves healthspan and lifespan in mice. Nat Commun.
2013;4:2192.

45. Alayev A, Berger SM, Holz MK. Resveratrol as a novel treatment for diseases with $\mathrm{mTOR}$ pathway hyperactivation. Ann N Y Acad Sci. 2015;1348(1):116-123.

46. Parmigiani A, et al. Sestrins inhibit mTORC1 kinase activation through the GATOR complex. Cell Rep. 2014;9(4):1281-1291.

47. Tomic T, et al. Metformin inhibits melanoma development through autophagy and apoptosis mechanisms. Cell Death Dis. 2011;2:e199.

48. Lehraiki A, et al. Inhibition of melanogenesis by the antidiabetic metformin. J Invest Dermatol. 2014;134(10):2589-2597.

49. Lee JH, et al. Sestrin as a feedback inhibitor of TOR that prevents age-related pathologies. Science. 2010;327(5970):1223-1228.

50. Lee JH, et al. Maintenance of metabolic homeostasis by Sestrin2 and Sestrin3. Cell Metab. 2012;16(3):311-321.

51. Wolfson RL, et al. Sestrin2 is a leucine sensor for the mTORC1 pathway. Science. 2016;351(6268):43-48.

52. Peng M, Yin N, Li MO. Sestrins function as guanine nucleotide dissociation inhibitors for Rag GTPases to control mTORC1 signaling. Cell. 2014;159(1):122-133.

53. Chantranupong L, et al. The Sestrins interact with GATOR2 to negatively regulate the aminoacid-sensing pathway upstream of mTORC1. Cell Rep. 2014;9(1):1-8.

54. Legro RS, et al. Clomiphene, metformin, or both for infertility in the polycystic ovary syndrome. N Engl JMed. 2007;356(6):551-566.

55. Cai H, et al. Cancer chemoprevention: Evidence of a nonlinear dose response for the protective effects of resveratrol in humans and mice. $\mathrm{Sci}$ Transl Med. 2015;7(298):298ra117.

56. Baur JA, et al. Resveratrol improves health and survival of mice on a high-calorie diet. Nature. 2006;444(7117):337-342.

57. Cantó $\mathrm{C}$, et al. Interdependence of AMPK and SIRT1 for metabolic adaptation to fasting and exercise in skeletal muscle. Cell Metab. 2010;11(3):213-219.

58. Dasgupta B, Milbrandt J. Resveratrol stimulates AMP kinase activity in neurons. Proc Natl Acad Sci U S A. 2007;104(17):7217-7222.

59. Park CE, et al. Resveratrol stimulates glucose transport in $\mathrm{C} 2 \mathrm{C} 12$ myotubes by activating AMP-activated protein kinase. Exp Mol Med. 2007;39(2):222-229.

60. Um JH, et al. AMP-activated protein kinase-deficient mice are resistant to the metabolic effects of resveratrol. Diabetes. 2010;59(3):554-563.

61. Pearson KJ, et al. Resveratrol delays age-related deterioration and mimics transcriptional aspects of dietary restriction without extending life span. Cell Metab. 2008;8(2):157-168.

62. Straley ME, Togher KL, Nolan AM, Kenny LC, O'Keeffe GW. LPS alters placental inflammatory and endocrine mediators and inhibits fetal neurite growth in affected offspring during late gestation. Placenta. 2014;35(8):533-538.

63. Elovitz MA, Brown AG, Breen K, Anton L, Maubert $\mathrm{M}$, Burd I. Intrauterine inflammation, insufficient to induce parturition, still evokes fetal and neonatal brain injury. Int J Dev Neurosci. 2011;29(6):663-671. 